\title{
Hard X-ray emission of the luminous infrared galaxy NGC 6240 as observed by NuSTAR
}

\author{
S. Puccetti ${ }^{1,2}$, A. Comastri ${ }^{3}$, F. E. Bauer ${ }^{4,5,6,7}$, W. N. Brandt ${ }^{8,9,10}$, F. Fiore ${ }^{2}$, F. A. Harrison ${ }^{11}$, B. Luo ${ }^{8,9}$, D. Stern ${ }^{12}$, \\ C. M. Urry ${ }^{13}$, D. M. Alexander ${ }^{14}$, A. Annuar ${ }^{14}$, P. Arévalo ${ }^{4,15}$, M. Baloković ${ }^{11}$, S. E. Boggs ${ }^{16}$, M. Brightman ${ }^{11}$, \\ F. E. Christensen ${ }^{17}$, W. W. Craig ${ }^{16,18}$, P. Gandhi ${ }^{14,19}$, C. J. Hailey ${ }^{20}$, M. J. Koss ${ }^{21}$, S. La Massa ${ }^{13}$, A. Marinucci ${ }^{22}$, \\ C. Ricci ${ }^{4,5}$, D. J. Walton ${ }^{12,11}$, L. Zappacosta ${ }^{2}$, and W. Zhang ${ }^{23}$
}

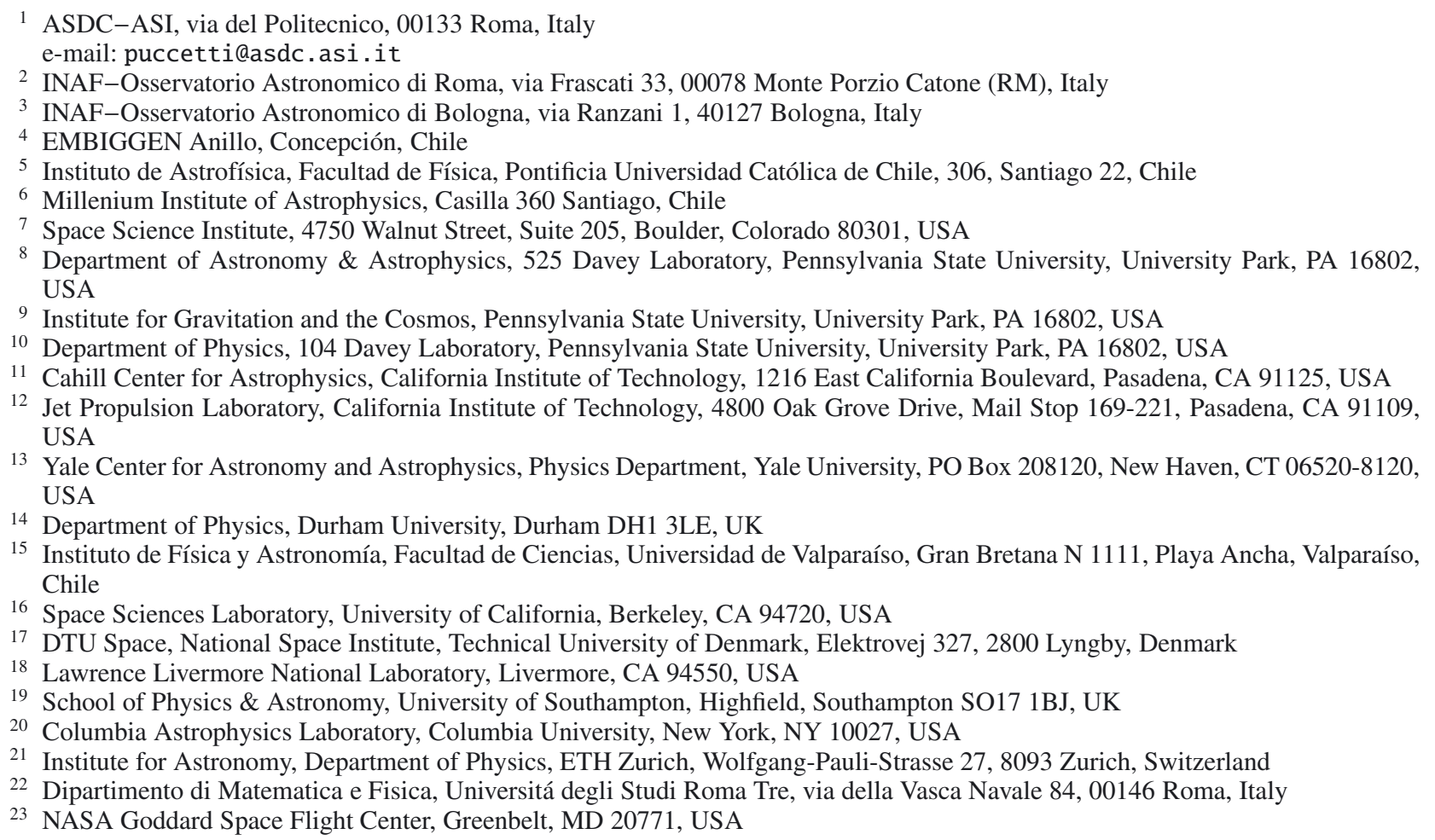

Received 14 August 2015 / Accepted 14 October 2015

\begin{abstract}
We present a broadband $(\sim 0.3-70 \mathrm{keV})$ spectral and temporal analysis of NuSTAR observations of the luminous infrared galaxy NGC 6240 combined with archival Chandra, XMM-Newton, and BeppoSAX data. NGC 6240 is a galaxy in a relatively early merger state with two distinct nuclei separated by $\sim 1$ ". 5 . Previous Chandra observations resolved the two nuclei and showed that they are both active and obscured by Compton-thick material. Although they cannot be resolved by NuSTAR, we were able to clearly detect, for the first time, both the primary and the reflection continuum components thanks to the unprecedented quality of the NuSTAR data at energies $>10 \mathrm{keV}$. The NuSTAR hard X-ray spectrum is dominated by the primary continuum piercing through an absorbing column density which is mildly optically thick to Compton scattering $\left(\tau \simeq 1.2, N_{\mathrm{H}} \sim 1.5 \times 10^{24} \mathrm{~cm}^{-2}\right)$. We detect moderately hard X-ray $(>10 \mathrm{keV}$ ) flux variability up to $20 \%$ on short $(15-20 \mathrm{ks})$ timescales. The amplitude of the variability is largest at $\sim 30 \mathrm{keV}$ and is likely to originate from the primary continuum of the southern nucleus. Nevertheless, the mean hard X-ray flux on longer timescales (years) is relatively constant. Moreover, the two nuclei remain Compton-thick, although we find evidence of variability in the material along the line of sight with column densities $N_{\mathrm{H}} \leq 2 \times 10^{23} \mathrm{~cm}^{-2}$ over long $(\sim 3-15 \mathrm{yr})$ timescales. The observed X-ray emission in the NuSTAR energy range is fully consistent with the sum of the best-fit models of the spatially resolved Chandra spectra of the two nuclei.
\end{abstract}

Key words. galaxies: active - galaxies: individual: NGC 6240 - X-rays: galaxies

\section{Introduction}

Galaxy mergers represent a crucial phase in most scenarios of galaxy formation. According to models and simulations (e.g.,
Springel et al. 2005; Di Matteo et al. 2005; Hopkins \& Elvis 2010), during the mergers of massive gas-rich galaxies, cold gas is destabilized and can rapidly form stars and will ultimately 
feed the active galactic nucleus (AGN). The relative importance of starbursts and AGNs is not known, nor are their associated timescales. Disentangling the contribution of these components (e.g., finding signatures of positive/negative feedback) in merging systems is crucial for comparing the observations with model predictions. In this respect NGC 6240 plays a central role as one of the nearest $\left(z=0.02448, D_{\mathrm{L}}=111.2 \mathrm{Mpc}\right.$, $D_{\mathrm{A}}=105.9 \mathrm{Mpc}$ ) luminous infrared galaxies observed in a relatively early merger state (Tacconi et al. 1999; Tezca et al. 2000; Bush et al. 2008; Engel et al. 2010; Medling et al. 2011). The infrared luminosity $\left(L_{\mathrm{IR}} \sim 10^{11.94} L_{\odot}=3.4 \times 10^{45} \mathrm{erg} \mathrm{s}^{-1}\right.$, Wright et al. 1984; Sanders et al. $2003^{1}$ ) is at the boundary between luminous infrared galaxies (LIRGs: $\left.L_{\mathrm{IR}}=\left(10^{11}-10^{12}\right) L_{\odot}\right)$ and ULIRGs (ultra-luminous infrared galaxies: $L_{\mathrm{IR}} \geq 10^{12} L_{\odot}$ ) and implies a high star formation rate $\left(S F R=61 \pm 30 M_{\odot} \mathrm{yr}^{-1}\right.$ from Yun \& Carilli 2002, through fitting of the far-infrared spectral energy distribution). Owing to the SFR which is high for its mass (e.g., Santini et al. 2009), NGC 6240 is classified as a starburst galaxy.

The optical emission-line spectrum (Fosbury \& Wall 1979; Zasov \& Karachentsev 1979; Fried \& Schulz 1983; Morris \& Ward 1988; Keel 1990; Heckman et al. 1987; Veilleux et al. 1995; Schmitt et al. 1996; Rafanelli et al. 1997) is dominated by LINER-like line ratios over the central $\sim 10 \mathrm{kpc}$. This spectrum is preferentially associated with shocks produced by cloud-cloud collisions in the merging system and not with a central photoionizing AGN continuum (see, e.g., Fosbury \& Wall 1979; Fried \& Schulz 1983). Nevertheless, a few high-excitation features in IR spectra and in optical HST narrowband images indicate possible weak AGN signatures in the southern region of the galaxy (Rafanelli et al. 1997).

The definite presence of an obscured AGN in NGC 6240 has been known since the first ASCA hard X-ray observations showed that a reflection spectrum provides the best fit below $10 \mathrm{keV}$ (Iwasawa \& Comastri 1998). The primary emission obscured by a column density of about $N_{\mathrm{H}} \sim 2 \times 10^{24} \mathrm{~cm}^{-2}$ emerges only at higher energies and was originally detected by the PDS instrument on board BeppoSAX (Vignati et al. 1999). The nuclear intrinsic bolometric luminosity is in the quasar regime $\left(L_{\mathrm{bol}} \sim 10^{45} \mathrm{erg} \mathrm{s}^{-1}\right.$, Vignati et al. 1999; Ikebe et al. 2000; Lira et al. 2002; Boller et al. 2003).

The presence of both a transmitted primary absorbed continuum and a cold reflection component is still under debate. The results obtained by the broadband spectral analysis up to energies of 100-200 keV (Vignati et al. 1999; Ikebe et al. 2000) are controversial. Vignati et al. (1999) clearly detect the transmitted primary absorbed continuum, but the presence of the cold reflection component is not significant. On the other hand, Ikebe et al. (2000), using RXTE data, obtained an equally good fit with either a reflection-dominated spectrum or with a reflected plus transmitted model. Therefore, the presence of a primary absorbed continuum is not certain from their spectral analysis. However, Ikebe et al. (2000) concluded that both components are needed because the intrinsic power law photon index is unusually flat $(\Gamma=1.26 \pm 0.13)$ in the pure reflection-dominated model.

Historically, NGC 6240 has shown moderate X-ray variability on month/year timescales at energies larger than $\sim 15 \mathrm{keV}$ $(22 \pm 7 \%, 54 \pm 18 \%$, and $4 \pm 3 \%$ in the $14-100,14-24$, and 35-100 keV Swift BAT energy bands, respectively; Soldi et al. 2014), while at softer energies it has not exhibited significant variability (Komossa et al. 1998; Netzer et al. 2005).

\footnotetext{
${ }^{1} L_{\mathrm{IR}}$ is the $8-1000 \mu \mathrm{m}$ luminosity according to the cosmology used in this paper.
}

NGC 6240 is the result of the merging of two smaller galaxies, which formed a larger galaxy with two distinct nuclei with an angular separation of $\sim 1^{\prime \prime} .5(\sim 0.7 \mathrm{kpc})$. The two nuclei are observed in near-infrared (e.g., Fried \& Schultz 1983), in the radio (e.g., Condon et al. 1982), and in optical (e.g., Rafanelli et al. 1997) images.

At X-ray wavelengths and thanks to its unsurpassed X-ray angular resolution (FWHM $\sim 0$.'5), Chandra identified that the two nuclei are both active and both exhibit highly obscured X-ray spectra typical of Compton-thick AGNs (Komossa et al. 2003). The southern nucleus is also brighter in X-rays than the northern nucleus. The observed $0.1-10 \mathrm{keV}$ luminosities of the two nuclei are $1.9 \times 10^{42} \mathrm{erg} \mathrm{s}^{-1}$ and $0.7 \times 10^{42} \mathrm{erg} \mathrm{s}^{-1}$ (Komossa et al. 2003). The southern nucleus is also brighter based on 3-5 $\mu \mathrm{m}$ luminosity (see, e.g., Mori et al. 2014; Risaliti et al. 2006).

The black hole mass of the southern nucleus, obtained via high-resolution stellar kinematics, is $(0.84-2.2) \times 10^{9} M_{\odot}$ (Medling et al. 2011). The mass of the northern nucleus, if it follows the $M_{\mathrm{BH}}-\sigma$ relation (Tremaine et al. 2002), is $(1.4 \pm 0.4) \times 10^{8} M_{\odot}$ (Engel et al. 2010). Owing to the large scatter in the relation, this value is subject to systematic uncertainties that are much larger than the measurement errors.

The two nuclei are surrounded by extended X-ray emission modeled as a multitemperature plasma; the temperature and column density increase toward the central regions and the metal abundances are higher than solar $\left(\frac{Z}{Z_{\odot}} \sim 2-2.5-\right.$ Netzer et al. 2005; $\frac{Z}{Z_{\odot}}=10$ - Boller et al. 2003; Wang et al. 2014). The hot gas, associated with highly ionized Fe XXV - emitting gas, peaks centrally at the southern nucleus, with $\sim 30 \%$ of the emission originating outside the nuclear region. Its temperature $\left(T \sim 7 \times 10^{7} \mathrm{~K}\right)$ indicates the presence of fast shocks with velocities on the order of $\sim 2200 \mathrm{~km} \mathrm{~s}^{-1}$ (Feruglio et al. 2013a; Wang et al. 2014). The energetics and the iron mass in the hot phase suggest that the fast shocks are due to a starburstdriven wind expanding into the ambient dense gas. Nevertheless, Wang et al. (2014) were not able to rule out additional energy injection in the circumnuclear region such as heating from an AGN outflow. High-resolution mapping of the $\mathrm{CO}(1-0)$ transition (Feruglio et al. 2013b) revealed the presence of a molecular outflow with velocity $\sim(200-500) \mathrm{km} \mathrm{s}^{-1}$ originating from the southern nucleus, likely driven by both supernova winds and radiation from the AGN. Alternatively, the $\mathrm{CO}(1-0)$ molecular gas condenses within the outflowing gas because the velocity difference between ionized and molecular outflows would lead to severe shredding (Veilleux et al. 2013). The coldest and outermost plasma forms a soft X-ray halo extending up to $\sim 1.5$ from the two nuclei with subsolar metal abundances $\left(\frac{Z}{Z_{\odot}}=0.1-0.5-\right.$ Nardini et al. 2013).

In this paper, we discuss the broadband $(\sim 0.3-70 \mathrm{keV})$ spectral and temporal properties of NuSTAR observations of NGC 6240 combined with archival XMM-Newton, Chandra, and BeppoSAX data. In Sect. 2, we describe the NuSTAR and archival observations. In Sect. 3 we discuss the variability properties. Section 4 presents the broadband spectral analysis, and in Sect. 5 we compare the individual Chandra spectra of the two nuclei with the NuSTAR spectrum of the galaxy as a whole. The results and conclusions are presented in Sects. 6 and 7, respectively.

Throughout this paper, we adopt a $\Lambda \mathrm{CDM}$ cosmology with $\Omega_{\mathrm{m}}=0.27, \Omega_{\Lambda}=0.73$, and $H_{0}=67.3 \mathrm{~km} \mathrm{~s}^{-1} \mathrm{Mpc}^{-1}$ (Planck Collaboration XVI 2014). 
Table 1. NuSTAR NGC 6240 observation log.

\begin{tabular}{lcccccc}
\hline \hline Observation ID $^{a}$ & $\begin{array}{c}\text { RA_PNT }^{b} \\
(\mathrm{deg})\end{array}$ & $\begin{array}{c}\text { Dec_PNT } \\
(\mathrm{deg})\end{array}$ & $\begin{array}{c}\text { Exposure }^{d} \\
(\mathrm{ks})\end{array}$ & Start date $^{e}$ & $\begin{array}{c}\text { Rate }^{f} \\
(\mathrm{cts} / \mathrm{s})\end{array}$ & Background $^{g}$ \\
\hline 60002040002 & 253.2528 & 2.4308 & 30.8 & 2014-Mar-30T13:41:07 & $0.159 \pm 0.002$ & $7.6 \%$ \\
\hline
\end{tabular}

Notes. ${ }^{(a)}$ Observation identification number; ${ }^{(b)}$ Right Ascension of the pointing; ${ }^{(c)}$ declination of the pointing; ${ }^{(d)}$ total net exposure time; ${ }^{(e)}$ start date and time of the observation; ${ }^{(f)}$ mean value of the net count rate in the circular source extraction region with $75^{\prime \prime}$ radius in the energy range $3-78.4 \mathrm{keV} ;{ }^{(g)}$ background percentage in the circular source extraction region with $75^{\prime \prime}$ radius and in the energy range $3-78.4 \mathrm{keV}$.

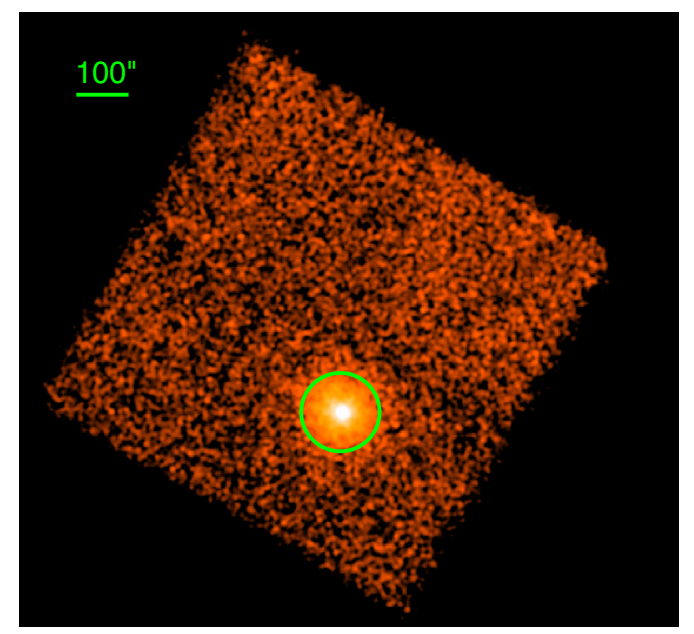

Fig. 1. 3-78.4 keV NuSTAR image for the FPMA module. The image was smoothed with a Gaussian filter with $\sigma=1.5$ pixels $(\sim 3$.' 6$)$. The green circle is centered on the peak of the emission and has a radius of $75^{\prime \prime}$.

\section{X-ray observations and data reduction}

\subsection{NUSTAR}

NuSTAR consists of two focal plane modules, FPMA and FPMB; it is sensitive at $3-78.4 \mathrm{keV}$ and has a field of view (FOV) at $10 \mathrm{keV}$ of $10^{\prime}$ (Harrison et al. 2013). The observatory has a $18^{\prime \prime}$ FWHM with a half-power diameter of $58^{\prime \prime}$. We analyzed the NuSTAR observation of NGC 6240 performed in March 2014. The observation log is given in Table 1.

The raw events files were processed using the NuSTAR Data Analysis Software package v. 1.4.1 (NuSTARDAS) ${ }^{2}$. Calibrated and cleaned event files were produced using the calibration files in the NuSTAR CALDB (20150225) and standard filtering criteria with the nupipeline task. We used the nuproducts task included in the NuSTARDAS package to extract the NuSTAR source and background spectra using the appropriate response and ancillary files. We extracted spectra and light curves in each focal plane module (FPMA and FPMB) using circular apertures of radius $75^{\prime \prime}$, corresponding to $\sim 80 \%$ of the encircled energy, centered on the peak of the emission in the $3-78.4 \mathrm{keV}$ data (see Fig. 1). Background spectra were extracted using sourcefree regions on the same detector as the source. As shown in Table 1 , the background count-rates are a low fraction $(<8 \%)$ of the source count-rates.

The spectra were binned according to two criteria: (i) following the energy resolution multiplied by a factor $\sim 0.4$ at all energies, when possible and (ii) requiring a signal-to-noise ratio $(\mathrm{S} / \mathrm{N})>4.5$. Spectral fitting was performed in the NuSTAR

\footnotetext{
2 http://heasarc.gsfc.nasa.gov/docs/nustar/analysis/ nustar_swguide.pdf
}

band alone and simultaneously with lower energy X-ray data (see below).

\subsection{Chandra}

Chandra, thanks to its unsurpassed X-ray angular resolution (FWHM $\sim 0.5)$, is an excellent complement to NuSTAR for analyzing the two nuclei and nearby regions and for studying possible contamination from sources that cannot be resolved by NuSTAR.

We analyzed four archival Chandra observations collected in 2001 and 2011 (see Table 2) using the Chandra Interactive Analysis Observations (CIAO) software (v4.5; Fruscione et al. 2006) and the standard data reduction procedures. The specextract task was used to extract the spectra. The background spectra were extracted from source-free regions at distances greater than about 1.5 from the nuclei of the galaxy in order to avoid contamination from the X-ray diffuse emission, which is still seen on scales of $1^{\prime}$ (see Nardini et al. 2013). The spectra were binned to have at least 25 total counts per bin. The nuclei showed negligible pile-up (less than a few percentage points). For the final spectral analysis, we used only the two ACIS-S observations (2001 and 2011) due to calibration uncertainties above $5 \mathrm{keV}$ of HETG 0th order ACIS-S spectra.

To avoid a loss of angular sensitivity, we registered the astrometry of the ACIS-S 2001 data to the ACIS-S 2011 data using the X-ray positions of bright common point-like sources. We found that the ACIS-S 2001 right ascension astrometric correction is -0 !' 1 , while the declination astrometric correction is negligible (i.e., 0.'01).

From the analysis of the Chandra images (see upper panel of Fig. 2), using an aperture photometry technique (see, e.g., Puccetti et al. 2009), we identified nine serendipitous point sources with $S / N \geq 2$ in the $0.3-8 \mathrm{keV}$ band within or very near the area corresponding to the NuSTAR extraction region (i.e., a circle with a radius of $75^{\prime \prime}$ ). We co-added the source spectra, background spectra, and ancillary files of the nine sources with addascaspec FTOOLS v.6.13, leaving the original exposure time of a single source in the final co-added spectrum. The total $0.3-8 \mathrm{keV}$ flux of these nine sources (green spectrum in the lower panel of Fig. 2) is definitely negligible in comparison to the flux in the NuSTAR extraction region (yellow spectrum in the lower panel of Fig. 2); it is fitted by a blackbody of $k T \sim 0.8 \mathrm{keV}$ and there is no evidence of any iron line emission. Therefore, the co-added spectrum of the nine brightest "contaminants" is consistent with a typical spectrum of ultra-luminous X-ray sources (ULXs), which turns over very quickly at about $10 \mathrm{keV}$ as discovered by NuSTAR (see, e.g., Walton et al. 2015). For these reasons we conclude that any contamination by close point-like sources in the NuSTAR spectrum is negligible.

The second step was to extract the Chandra spectra of the whole galaxy (i.e., the combination of the two nuclei, extended emission, and serendipitous sources) using a circular region of 

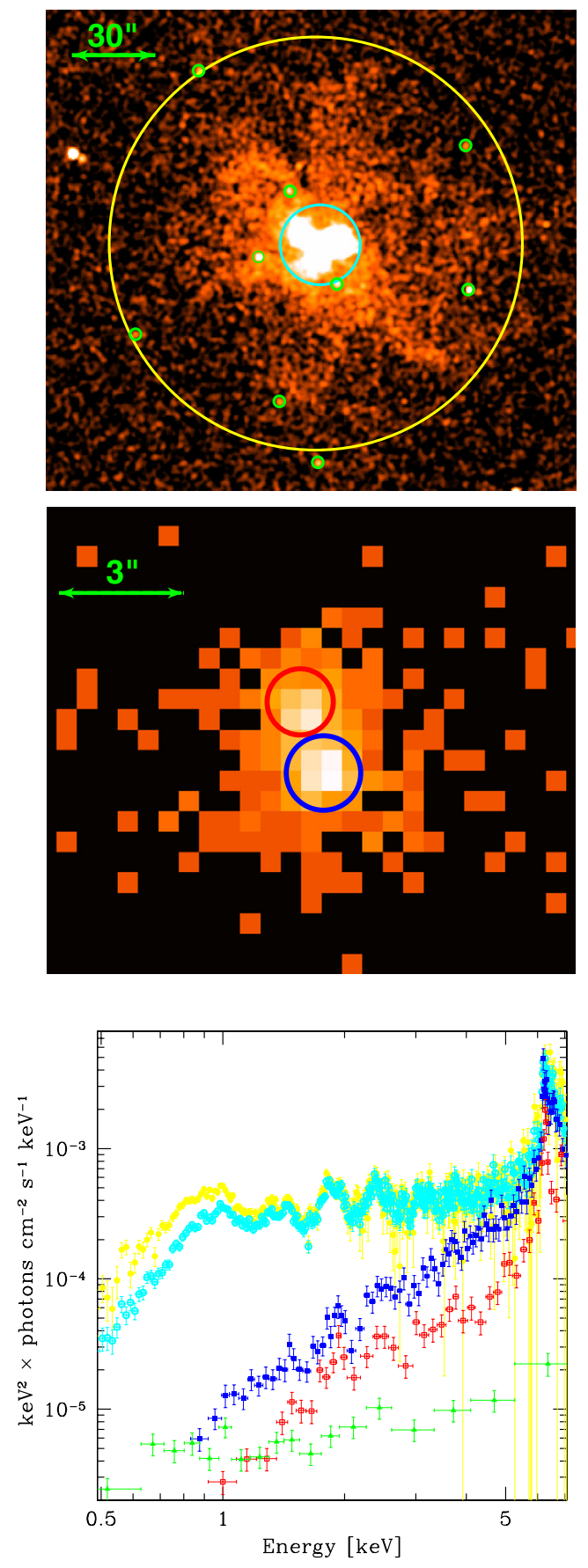

Fig. 2. Upper panel: $0.3-8 \mathrm{keV}$ image mosaic of the 2001 and 2011 Chandra observations (see Table 2). The image was smoothed with a Gaussian filter with $\sigma=1.5$ pixels $(\sim 0$.'75). The big yellow circle (75" radius) marks the NuSTAR source extraction region; the cyan circle (14". 5 radius) marks the Chandra source extraction region; the small green circles ( $2^{\prime \prime}$ radius) mark the nine serendipitous point sources with $S / N \geq 2$ in the $0.3-8 \mathrm{keV}$ band within/very near the area corresponding to the NuSTAR extraction region. Middle panel: $6-6.5 \mathrm{keV}$ image mosaic of the two Chandra observations, zoomed into the center of the galaxy: the southern and northern nuclei are shown by blue and red circles, respectively. Lower panel: Chandra spectra unfolded with the instrument response of observation 12713 (see Table 2). The yellow, cyan, blue, and red spectra are extracted in the regions marked with the same color in the upper and middle panels. The green spectrum represents the contamination spectrum by the nearby bright sources detected in the NuSTAR source extraction region. This contamination is negligible in the NuSTAR extraction region, whose spectrum is shown in yellow. radius 14 .'5 to maximize the $\mathrm{S} / \mathrm{N}$ of the spectra. In this way we excluded most of the very soft X-ray emission in the halo of NGC 6240 ( $\leq 2 \mathrm{keV}$, see Nardini et al. 2013). This component is not detectable in the energy band covered by NuSTAR, which has a low energy limit at $3 \mathrm{keV}$. This is clearly seen in the comparison of the cyan and yellow spectra in the lower panel of Fig. 2.

Finally, we verified that the whole galaxy did not show significant variations during the four Chandra observations (see Fig. 3). We used the $\chi^{2}$ statistics with a threshold probability of $2 \%$ to test the hypothesis of constant light curves. The $0.3-8 \mathrm{keV}$ light curve of the whole galaxy is consistent with it being constant between the two ACIS-S and the two ACIS-S HETG observations. The $6.4-8 \mathrm{keV}$ count rates are only $\sim 0.3 \%$ and $\sim 10 \%$ of those in the full energy band, for the ACIS-S and the zeroorder ACIS-S HETG, respectively. For this reason small amplitude hard X-ray variability is difficult to detect in the full energy range; therefore we also verified that the 6.4-8 keV count rates are statistically constant. The ACIS-S and ACIS-S HETG spectra are fully consistent with each other (see Fig. 4) confirming that the variability in the whole galaxy is not significant. Therefore, in order to improve the statistics for the final spectral analysis we used the co-added spectra of the two ACIS-S (2001 and 2011) observations and we also combined the corresponding background spectra, response, and ancillary files. We used addascaspec FTOOLS v.6.13, which combines spectra and normalized backgrounds according to the method explained in the ASCA ABC guide ${ }^{3}$.

In addition to the spectrum of the whole galaxy, we also used the ACIS-S spectra of each of the two individual nuclei (see Komossa et al. 2003). The spectra were extracted using circular regions centered on $\mathrm{RA}=16: 52: 58.896, \mathrm{Dec}=+02: 24: 03.36$ and $\mathrm{RA}=16: 52: 58.922$, $\mathrm{Dec}=+02: 24: 05.03$ and with a radius of 0.9 for the southern nucleus (the brightest one) and 0.8 for the northern nucleus, respectively (blue and red circles and spectra in the middle and bottom panel of Fig. 2, respectively).

The $0.3-8 \mathrm{keV}$ light curves of the southern and northern nucleus are consistent with being constant between the two ACIS$\mathrm{S}$ and the two ACIS-S HETG observations (see Fig. 3). We also searched for variability in the two sub-bands: $0.3-6.4 \mathrm{keV}$ and $6.4-8 \mathrm{keV}$. We found variability only in the $6.4-8 \mathrm{keV}$ light curve of the northern nucleus in the ACIS-S 2011 observation $\left(\chi_{v}^{2}=1.84\right.$ with probability of false positive $\leq 0.6 \%$, see bottom panels of Fig. 3). Nevertheless, the count rate variations are small and the median values of the count rates of the two ACIS$\mathrm{S}$ observations are fully consistent (i.e., $(0.011 \pm 0.001) \mathrm{cts} / \mathrm{s})$. Therefore, we also used the co-added spectra of the two ACIS-S (2001 and 2011) observations for the nuclei. We note that the 0.3-8 light curve of the northern nucleus of ACIS-S 2011 shows a systematic residual with respect to a fit with a constant in the time interval 100-120 ks in Fig. 3, which could be due to the $6.4-8 \mathrm{keV}$ variability.

\subsection{XMM-Newton}

In the spectral analysis we also considered six XMM-Newton observations with a nonzero exposure time after background cleaning, obtained between September 2000 and August 2003 (see Table 2). The high spectral resolution of XMM-Newton at $\sim 6 \mathrm{keV}$ and the good counting statistics of the archival observations allow us to constrain the spectral shape and the intensity of the iron line complex. We reduced the data using the

http://heasarc.gsfc.nasa.gov/docs/asca/abc/ 

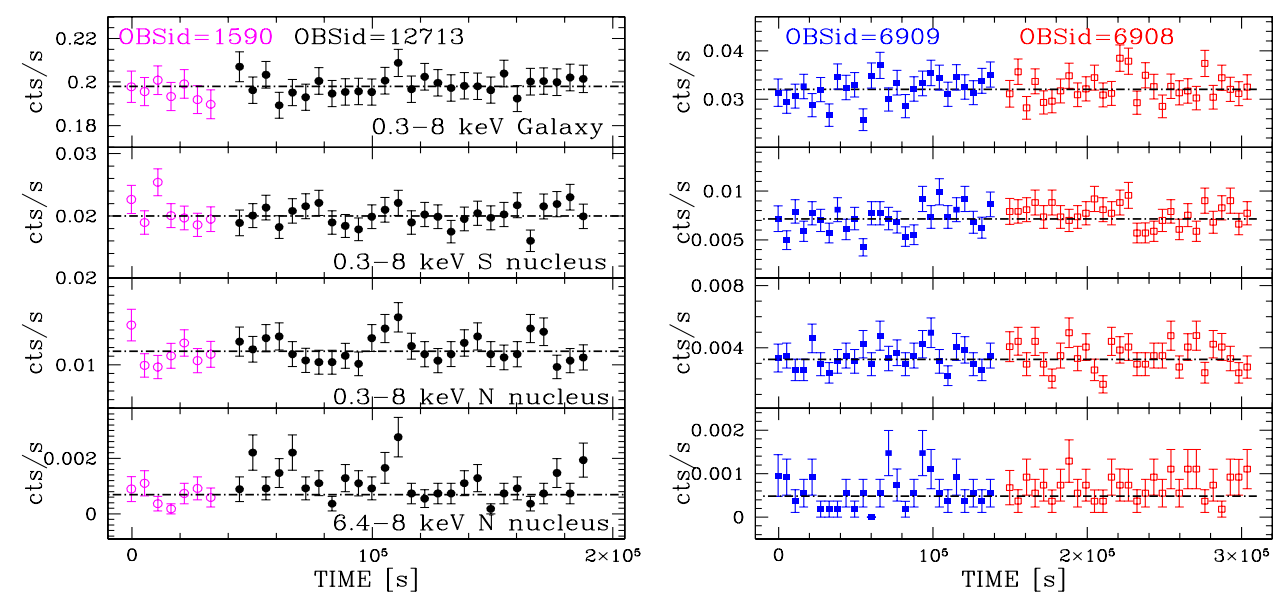

Fig. 3. Chandra backgroundsubtracted light curves in bins of $5500 \mathrm{~s}$. From top to bottom: $0.3-8 \mathrm{keV}$ count rates of the whole NGC 6240 galaxy, $0.3-8 \mathrm{keV}$ count rates of the southern nucleus, $0.3-8 \mathrm{keV}$ count rates of the northern nucleus, and $6.4-8 \mathrm{keV}$ count rates of the northern nucleus. The dot-dashed lines correspond to the values of the count rate weighted means. Left panels: the light curves of the two ACIS-S Chandra observations (see Table 2): 1590 (magenta open dots) and 12713 (black solid dots). Right panels: the light curves of the zero-order ACIS-S HETG Chandra data: 6909 (blue solid squares) and 6908 (red open squares). We note that the $X$-axis does not represent the real time.

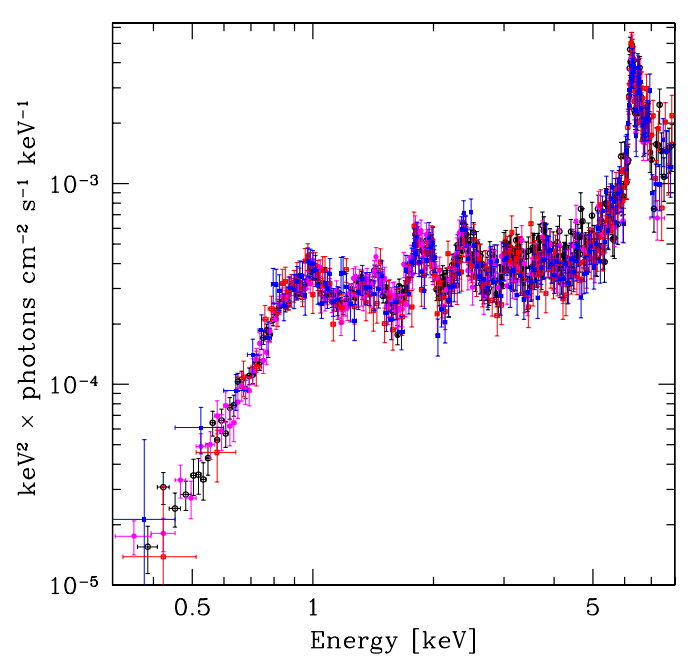

Fig. 4. Chandra spectra of the whole NGC 6240 galaxy, unfolded with the instrument response, extracted in the cyan region in the upper panel of Fig. 2. The colors represent the four observations (see Table 2). Since no significant spectral variability was present, we co-added the ACIS-S spectra.

Science Analysis Software (SAS ver. 14.0.0), following the standard method described in the data analysis threads and the $\mathrm{ABC}$ Guide to XMM-Newton Data Analysis ${ }^{4}$ Epochs of high background events were excluded; only events corresponding to pattern 0-12 for MOS1/MOS2 and pattern 0-4 for PN were used. From the analysis of the Chandra spectra, we found that the AGNs and hot gas in the galaxy are extended less than 15", thus the spectra were extracted using a circular region of radius $40^{\prime \prime}$, which corresponds to $\sim 90 \%$ of the encircled energy; this percentage is similar to the NuSTAR data, which includes all the galaxy emission at energy $\geq 2 \mathrm{keV}$. Extraction of the background spectra and the binning were done in a similar way to the NuSTAR analysis.

The whole NGC 6240 galaxy, as already pointed out by Netzer et al. (2005), does not exhibit significant spectral variability during the XMM-Newton observations (see also Fig. 5). Therefore, to improve the statistics, we considered the combination of all MOS1 and MOS2 spectra, and of the individual

\footnotetext{
4 http://xmm.esac.esa.int/sas/current/documentation/ threads/
}

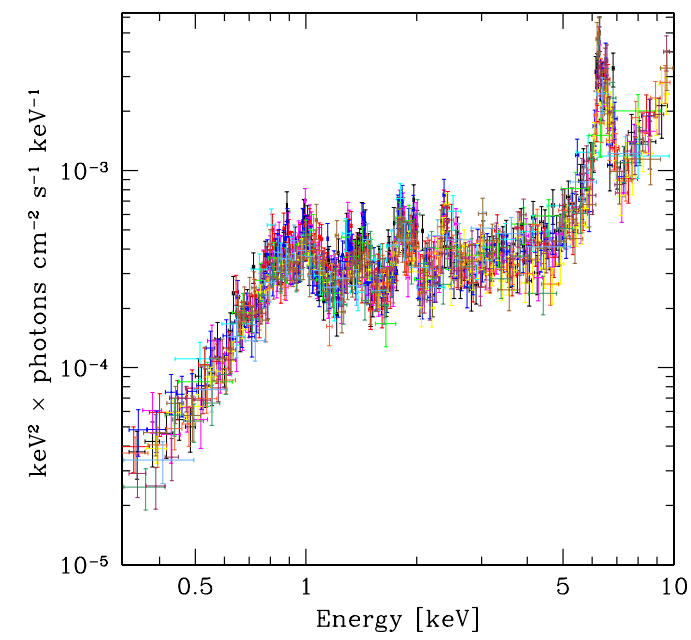

Fig. 5. XMM-Newton spectra of the whole NGC 6240 galaxy, unfolded with the instrument response. The colors represent the EPIC-PN and co-added EPIC-MOS1 and EPIC-MOS2 spectra for all the observations (see Table 2).

PN spectra. In the spectral analysis we used the XMM-Newton data between $2-10 \mathrm{keV}$ only to avoid a larger soft X-ray contribution in comparison with the Chandra spectra.

\subsection{BeppoSAX PDS data}

NGC 6240 was observed by BeppoSAX (Boella et al. 1997) on 14 August 1998 (Vignati et al. 1999) with the Low Energy Concentrator Spectrometer (LECS; 0.45-4 keV), the two Medium Energy Concentrator Spectrometers (MECS; 1.65-10.5 keV) and the Phoswitch Detector System (PDS; 15-200 keV, Frontera et al. 1997; see Table 2). Data products were retrieved from the BeppoSAX archive at the ASI Science Data Center ${ }^{5}$. The data were calibrated and cleaned using the SAXDAS software. The MECS/LECS event files were screened adopting standard pipeline selection parameters, and the spectra were extracted from 4' radii apertures, corresponding to $\sim 90 \%$ of the encircled energy. The two MECS units were combined after renormalizing to the MECS1. The PDS is a collimator instrument with a field of view of $\sim 103$ (FWHM). The

5 http://www.asdc.asi.it/mmia/index.php?mission=saxnfi 
Table 2. NGC 6240 archival data.

\begin{tabular}{|c|c|c|c|c|c|c|c|}
\hline Observatory & Observation $\mathrm{ID}^{a}$ & $\begin{array}{c}\text { Exposure }^{b} \\
(\mathrm{ks})\end{array}$ & Start Date ${ }^{c}$ & & & & Ref \\
\hline BeppoSAX & 5047600100 & 120 & 1998-Aug.-14 & & & & 1 \\
\hline Chandra ACIS-S & 1590 & 36.7 & 2001-Jul.-29T05:44:22 & & & & $2,3,4$ \\
\hline Chandra ACIS-S HETG & 6909 & 141.2 & 2006-May-11T01:41:48 & & & & $3,4,5$ \\
\hline Chandra ACIS-S HETG & 6908 & 157.0 & 2006-Мay-16T11:22:54 & & & & $3,4,5$ \\
\hline \multirow[t]{2}{*}{ Chandra ACIS-S } & 12713 & 145.4 & 2011-May-31T04:15:57 & & & & 3,4 \\
\hline & & & & $\mathrm{PN}^{d}$ & $\operatorname{MOS} 1^{d}$ & $\operatorname{MOS} 2^{d}$ & \\
\hline$X M M-N e w t o n$ & 0101640101 & 30.1 & 2000-Sep.-22T01:38:46 & 11.3 & 15.4 & 15.4 & 6,7 \\
\hline XMM-Newton & 0101640601 & 19.0 & 2002-Apr.-12T21:37:24 & 5.3 & 10.2 & 10.0 & 7 \\
\hline XMM-Newton & 0147420201 & 31.6 & 2003-Apr.-14T18:06:14 & 3.4 & 7.1 & 7.9 & 7 \\
\hline$X M M-N e w t o n$ & 0147420301 & 28.1 & 2003-Apr.-18T21:01:56 & 0 & 0 & 0 & 7 \\
\hline XMM-Newton & 0147420401 & 14.1 & 2003-Aug.-13T10:29:32 & 7.0 & 10.8 & 10.7 & 7 \\
\hline XMM-Newton & 0147420501 & 31.2 & 2003-Aug.-21T10:25:09 & 3.4 & 1.7 & 1.8 & 7 \\
\hline XMM-Newton & 0147420601 & 9.2 & 2003-Aug.-29T11:25:15 & 1.4 & 1.8 & 1.9 & 7 \\
\hline
\end{tabular}

Notes. ${ }^{(a)}$ Observation identification number; ${ }^{(b)}$ total net exposure time; ${ }^{(c)}$ start date and time of the observation interval; ${ }^{(d)}$ Good Time Interval after background cleaning.

References. 1) Vignati et al. (1999); 2) Komossa et al. (2003); 3) Nardini et al. (2013); 4) Wang et al. (2014); 5) Shu et al. (2011); 6) Boller et al. (2003); 7) Netzer et al. (2005).

PDS data were calibrated and cleaned following the standard "fixed Rise Time threshold" method for background rejection. Following the method used for the NuSTAR data, we estimate that for the MECS data, the contamination by the nearby bright sources is less than $10 \%$. We do not use the LECS data to avoid a larger soft X-ray contribution in comparison with data from other instruments used in this work.

\section{Light curve variability}

With a few exceptions (e.g., the Compton-thick to Compton-thin changes in NGC 1365, Risaliti et al. 2005, 2007), variability in Compton-thick AGN is extremely rare at energies below $10 \mathrm{keV}$. Constraining intrinsic variability in such obscured sources requires statistically good sampling of the 30-50 keV energy range where the primary continuum can arise. The analysis of the light curve in different energy ranges returns raw information on the origin of the variability (i.e., variations in Eddington ratio, column density, photon index of the primary continuum, reflection component). When data with good statistics are available, the following time/count rate resolved spectral analysis allows us to constrain the variable spectral parameters more accurately, and place constraints on the geometry of the reflecting/absorbing medium (see, e.g., NGC 4945 Puccetti et al. 2014; NGC 1068 Marinucci et al. 2016). Unfortunately, the statistics do not allow this detailed spectral analysis for NGC 6240.

The NuSTAR NGC 6240 observation lasted $\sim 60 \mathrm{ks}$, but the Earth occults the target for approximately half the orbit, yielding a net exposure time of $30.8 \mathrm{ks}$. We analyzed the NuSTAR light curves in bins of $5800 \mathrm{~s}$ ( $\sim 1$ satellite orbit). We used the $\chi^{2}$ and the Kolmogorov-Smirnov tests to verify the constancy of the light curves. A light curve is considered definitely variable if the probability of a false positive is $\leq 2 \%$ for at least one of the two tests.

The 3-60 keV light curve is slightly variable and shows a systematic trend of the residuals with respect to a fit with a constant (see Fig. 6). To investigate possible spectral variations, we analyzed the 3-10 keV and the 10-60 keV light curves separately. We found that the $3-10 \mathrm{keV}$ light curve is fully consistent with it being constant, but the $10-60 \mathrm{keV}$ light curve is not, showing variability up to $\sim 20 \%$. The $3-10 \mathrm{keV}$ light curve is not correlated with the $10-60 \mathrm{keV}$ at a confidence level larger than $99.99 \%$ using the Spearman rank correlation coefficient. In the three energy bands, the background level is on the order of $7 \%$ of the total count rate. Moreover, we verified that the source and background light curves are not correlated at a confidence level of $5 \%$.

To better analyze the hard X-ray variability, we analyzed NuSTAR light curves in three narrower energy ranges: 10-25, 25-35, and 35-60 keV. We found that the amplitude of the variations is largest in the $25-35 \mathrm{keV}$ energy band, changing by $\sim 40 \%$ on timescales of $\sim 15-20 \mathrm{ks}$. We also searched for hard X-ray variability on similarly short timescales using the archival BeppoSAX 15-200 keV PDS data. The PDS light curve is not constant and the largest amplitude variability is $\sim 50 \%$ on timescales of $\sim 20 \mathrm{ks}$ (see Fig. 7), fully consistent with the NuSTAR findings.

The 20-70 keV PDS mean flux, evaluated assuming a power-law model with photon index fixed to 1.7 , is a factor of $\sim 0.9$ the $20-70 \mathrm{keV}$ NuSTAR mean flux. This factor contains possible source variability and also variability due to the intercalibration uncertainty between the two instruments.

As shown in Sect. 2.2, we also searched for variability in the Chandra data in the hardest accessible energy range (6.4-8 keV) of the northern and the southern nuclei separately. The count rate of the 2011 ACIS-S observation of the northern nucleus is found to be variable by a factor of $\sim 1.3$ on timescales of $\sim 15 \mathrm{ks}$, while the southern nucleus is constant. In the two 2006 ACIS-S HETG observations we did not find statistically strong evidence of variability in the southern or in the northern nucleus. This could be due to the poorer statistics of the zero-order ACIS-S HETG data, in comparison to ACIS-S data (i.e., for the northern nucleus we had 50\% fewer counts in the ACIS-S HETG).

In the 6.4-8 keV energy range, the composed light curve of the northern and southern nuclei does not present the variability shown by the northern nucleus alone because the northern nucleus is a factor of $\sim 0.4$ fainter and the amplitude of its variations is small. This result is consistent with the absence of variability in the $6.4-10 \mathrm{keV}$ energy range in the XMM-Newton observations. 


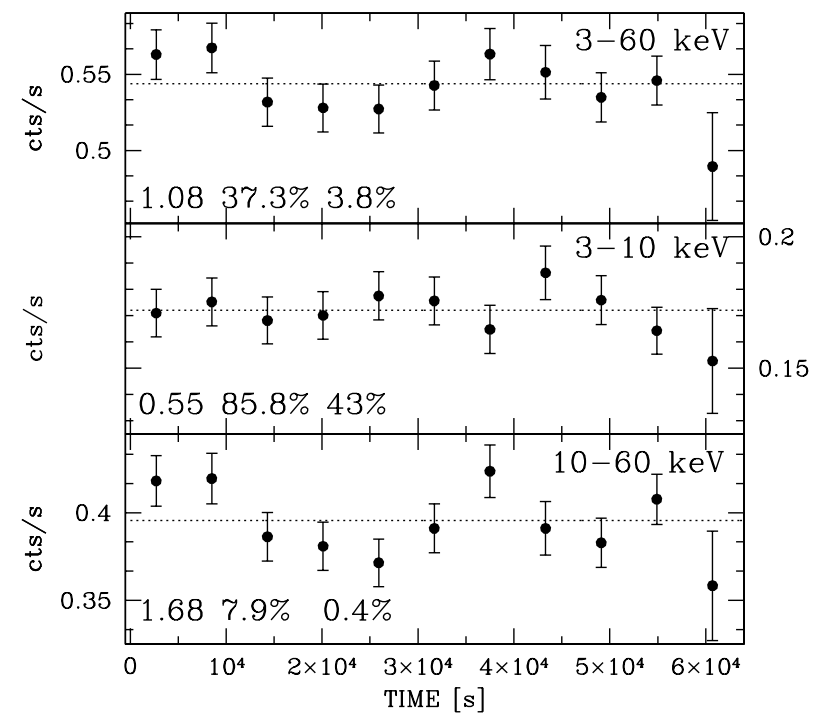

Fig. 6. NuSTAR light curves in bins of $5800 \mathrm{~s}$ ( $\sim 1$ satellite orbit). The count rates are the added values detected by the FPMA and FPMB modules corrected for livetime, PSF losses, and vignetting, but not for background. The dotted lines correspond to the values of the count rate weighted means. The numbers in the lower left corner of each panel indicate the values of $\chi_{v}^{2}$ for 10 degrees of freedom, and the false positive $\chi^{2}$ and Kolmogorov-Smirnov probabilities in the constant light curve hypothesis. From top to bottom: 3-60, 3-10, and 10-60 keV count rates.

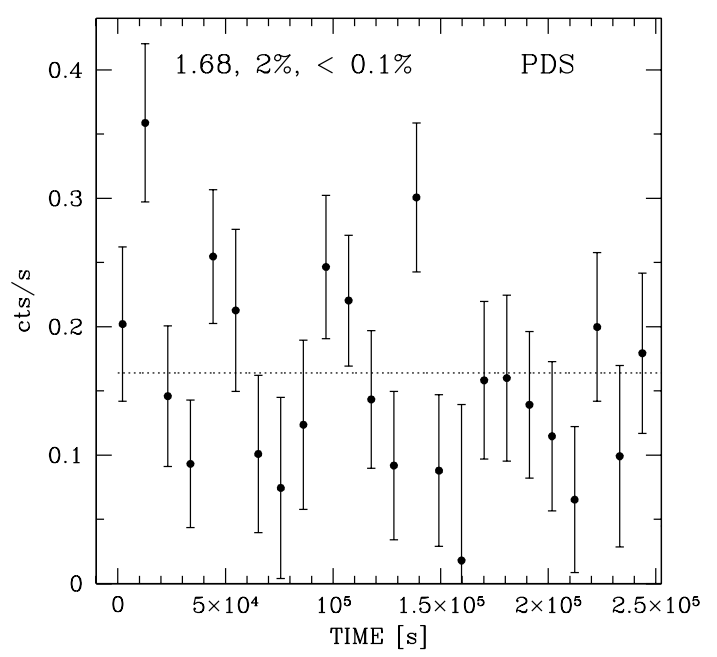

Fig. 7. BeppoSAX 15-200 keV PDS light curve in bins of $10.5 \mathrm{ks}$. The dotted line corresponds to the value of the count rate weighted mean. The numbers at the top indicate the values of $\chi_{v}^{2}$ for 23 degrees of freedom, and the false positive $\chi^{2}$ and Kolmogorov-Smirnov probabilities in the constant light curve hypothesis.

\section{Broadband spectral analysis}

\subsection{The model}

The extended X-ray emission at energies $<5 \mathrm{keV}$ in NGC 6240 is due to an absorbed multitemperature plasma. Both the temperature and the column density increase toward the central nuclei (Boller et al. 2003; Netzer et al. 2005; Wang et al. 2014). The cold-temperature plasma, which is the outermost, extends up to $\sim 1.5$ from the nuclei, has subsolar metal abundances $\left(\frac{Z}{Z_{0}}=0.1-0.5\right.$, see Nardini et al. 2013) and dominates at energies $\leq 1.5 \mathrm{keV}$. A medium-temperature plasma, which is less extended, is closer to the central nuclei. The hot-temperature plasma, which is dominant in the $\sim 2-5 \mathrm{keV}$ energy range, indicates the presence of fast shocks (Feruglio et al. 2013a; Wang et al. 2014, ACIS-S and HETG ACIS-S data) and is responsible for the Fe XXV line emission. While a scattered nuclear powerlaw would provide an equally good fit, the Fe XXV line emission is extended and spatially resolved by Chandra (Wang et al. 2014). Moreover, a dominant contribution to the Fe XXV from photoionization is ruled out through the comparison of the expected X-ray line ratios with the observed values (Netzer et al. 2005), unlike some non-LIRG AGN (e.g., NGC 1068, Bauer et al. 2015 and references therein; CIRCINUS, Arévalo et al. 2014 and references therein) where photoionization is dominant.

The X-ray spectrum of NGC 6240 shows strong emission lines of MgXII (1.47 keV), Si XIII (1.865 keV), Si XIV (2.006 keV), S XV (2.461 keV), and S XVI (2.622 keV) due to the hot thermal component, which are difficult to reproduce within the context of equilibrium models with solar metal abundance. Therefore, to reproduce these line ratios, the hot thermal component was modeled by the XSPEC APEC/MEKAL models with super-solar metal abundance $\left(\frac{Z}{Z_{0}} \sim 2-2.5\right.$ - Netzer et al. 2005; $\frac{Z}{Z_{0}}=10-$ Boller et al. 2003). A more empirical approach was followed by Wang et al. (2014), who proposed a model with a MEKAL component with solar abundances, plus several Gaussians to fit the residual lines. Alternatively, the observed strong emission lines are expected in nonequilibrium models, in which the ion distribution is broader than in thermal equilibrium models with the same physical conditions; in this context, Feruglio et al. (2013a) modeled the hot thermal component by a shock model with almost solar abundances. Based on these arguments, we attempted to model the hot thermal component with two different approaches: a shock model (i.e., XSPEC PSHOCK model, hereafter model A), and the usual MEKAL component (model B). As shown below, the solar chemistry can be used for model A, whereas model B requires super-solar metal abundances.

In the energy range $5-8 \mathrm{keV}$ the emission is mainly due to the two obscured AGNs in the center of NGC 6240 (Komossa et al. 2003). Both nuclei are best fitted with a hard, reflectiondominated spectrum and associated iron line complex (i.e., $\mathrm{FeK}_{\alpha}$ at $6.4 \mathrm{keV}, \mathrm{FeK}_{\beta}$ at $7.06 \mathrm{keV}$ rest-frame energies and the Compton shoulder).

In the NuSTAR band, the two nuclei cannot be spatially resolved, and their summed contribution in the hard X-ray band is modeled as follows: a) a primary continuum, transmitted through a high column density absorber, modeled by PLCABS (Yaqoob 1997); b) a reflection component, due to optically thick gas with infinite column density illuminated by a power continuum and modeled with PEXRAV (Magdziarz \& Zdziarski 1995). We investigate more physically realistic reflection models in Sects. 4.2 and 5.1. The photon indices of the primary and the reflected components are linked to the same value in the spectral fits. We also added three Gaussian lines to model the $\mathrm{FeK}_{\alpha}, \mathrm{FeK}_{\beta}$, and nickel $\mathrm{K}_{\alpha}$ at $7.47 \mathrm{keV}$. The $\mathrm{FeK}_{\alpha}$ and nickel $\mathrm{K}_{\alpha}$ are significant at $99.99 \%$ confidence level. The ratio between the normalization of the $\mathrm{FeK}_{\beta}$ and $\mathrm{FeK}_{\alpha}$ is fixed to the theoretical value (1:8.8; e.g., Palmeri et al. 2003). All the spectral components were absorbed by the Galactic column density along the line of sight (i.e., $4.87 \times 10^{20} \mathrm{~cm}^{-2}$; Kalberla et al. 2005) and redshifted using $z=0.02448$ (Downes et al. 1993). We used Anders \& Grevesse (1989) cosmic abundances. For the broadband analysis, we simultaneously fit the ACIS-S (0.3-8 keV), PN (2-10 keV), MOS (MOS1+MOS2) $(2-10 \mathrm{keV})$, FPMA (3-78.4 keV), and FPMB (3-78.4 keV) 
spectra of the whole galaxy. We introduced normalization factors to take into account the uncertain flux inter-calibration between different instruments. We fixed the normalization factor of FPMA to unity, leaving the other normalizations free to vary. As discussed above, we modeled the hot thermal components in two different ways. Therefore, we used two different models with the following XSPEC format

Model A:

CONSTANT $\times$ WABS $_{\text {Gal }} \times($ PLCABS + PEXRAV +ZGAUSS + ZGAUSS +

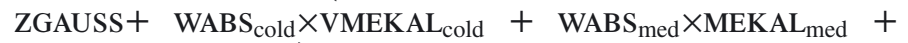
WABS $_{\text {hot }} \times$ PSHOCK $_{\text {hot }}$ )

Model B:

CONSTANT $\times$ WABS $_{\text {Gal }} \times($ PLCABS + PEXRAV+ZGAUSS +ZGAUSS +

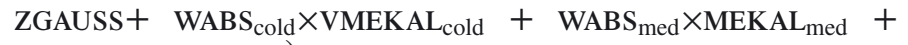
WABS $_{\text {hot }} \times$ MEKAL $_{\text {hot }}$ )

where the subscripts cold, med, and hot indicate the cold, medium-temperature, and hot thermal components, respectively.

\subsection{Spectral analysis results}

For both models $\mathrm{A}$ and $\mathrm{B}$, the cold thermal component requires a subsolar abundance $\left(\frac{Z}{Z_{\odot}}=0.2-0.5\right)$ of the $\alpha$ elements $(\mathrm{O}, \mathrm{Ne}, \mathrm{Mg}, \mathrm{Si}, \mathrm{S}, \mathrm{Ar})$ and a lower subsolar iron abundance $\left(\frac{\mathrm{Fe}}{\mathrm{Fe}_{\odot}}=0.1-0.2\right)$ at the $99.97 \%$ confidence level according to an F-test. This is consistent with Nardini et al. (2013). The absorbing column density for the cold thermal component $\left(N_{\mathrm{H}}=\right.$ $\left(0.12_{-0.06}^{+0.07}\right) \times 10^{22} \mathrm{~cm}^{-2}$ for model A) is slightly higher than, but consistent with, the Galactic value (i.e., $N_{\mathrm{H}}=0.05 \times 10^{22} \mathrm{~cm}^{-2}$ ). The column densities of the gas obscuring the medium $(k T \sim$ $0.9 \mathrm{keV}$ ) and the hot thermal components are similar, thus are linked together in the final fits $\left(N_{\mathrm{H}}=\left(0.97_{-0.09}^{+0.18}\right) \times 10^{22} \mathrm{~cm}^{-2}\right.$ for model A). This is higher than the column density of the cold thermal component. Model B requires super-solar metal abundances for the medium-temperature and hot components $\left(\frac{Z}{Z_{\odot}} \sim 2\right)$.

The best-fit parameters are listed in Table 3. Both models are fully consistent with the data $\left(\chi_{v}^{2} \sim 0.99\right)$; nevertheless, the quality of the fit for model B is slightly worse than for model A $\left(\Delta \chi^{2}=+13\right.$ for the same number of degrees of freedom). The super-solar metallicity of the hot thermal component required by model B may indicate that the model is oversimplified. Indeed multitemperature plasma, nonthermal plasma, and X-ray binaries could contribute to this component with different percentages (see, e.g., Bauer et al. 2015; Arévalo et al. 2014). On the other hand, the temperature of the shock in model $\mathrm{A}(k T=$ $7.3_{-1.4}^{+1.8} \mathrm{keV}$ ) is slightly higher than the temperature obtained by fitting only the XMM-Newton spectrum $(k T=5.5 \pm 1.5 \mathrm{keV}$; Boller et al. 2003; Netzer et al. 2005) or the Chandra data $(k T=$ $6.15 \pm 0.3 \mathrm{keV}$; Wang et al. 2014) with similar XSPEC models, but is consistent within the uncertainties. In fact, if the shock temperature is fixed at $6 \mathrm{keV}$ the best-fit solution is still statistically acceptable. On the basis of the above described physical arguments, we chose model $\mathrm{A}$ as the reference model. The broadband best-fit spectra residuals and model A are shown in Fig. 8.

The best-fit parameters of the absorbed power-law, describing the nuclear X-ray continuum emission of the two nuclei, are $\Gamma=1.75 \pm 0.08$ and $N_{\mathrm{H}}=\left(1.58_{-0.09}^{+0.10}\right) \times 10^{24} \mathrm{~cm}^{-2}$. The nuclear absorption-corrected $2-10 \mathrm{keV}$ luminosity is $\left(7.0_{-1.9}^{+2.6}\right) \times$ $10^{43} \mathrm{erg} \mathrm{s}^{-1}$; the reflection component in the $10-40 \mathrm{keV}$ energy range is on the order of $20 \%$ of the observed primary emission.
Table 3. Best-fitting parameters.

\begin{tabular}{|c|c|c|c|}
\hline Parameter & Model A & Model B & Units \\
\hline$N_{\mathrm{HGal}}{ }^{a}$ & 0.0487 & 0.0487 & $10^{22} \mathrm{~cm}^{-2}$ \\
\hline \multicolumn{4}{|c|}{ Primary and reflected components: PLCABS+PEXRAV+ZGAUSS+ZGAUSS+ZGAUSS } \\
\hline$N_{\text {Habs }}^{b}$ & $1.58_{-0.09}^{+0.10}$ & $1.63 \pm 0.10$ & $10^{24} \mathrm{~cm}^{-2}$ \\
\hline$\Gamma^{c}$ & $1.75 \pm 0.08$ & $1.82 \pm 0.07$ & \\
\hline Norm $_{\text {plcabs }}{ }^{d}$ & $136_{-36}^{+51}$ & $156_{-41}^{+58}$ & $10^{4} \mathrm{ph} \mathrm{keV}^{-1} \mathrm{~cm}^{-2} \mathrm{~s}^{-1}$ \\
\hline Norm $_{\text {pexrav }}{ }^{e}$ & $16 \pm 6$ & $36_{-6}^{+7}$ & $10^{4} \mathrm{ph} \mathrm{keV}^{-1} \mathrm{~cm}^{-2} \mathrm{~s}^{-1}$ \\
\hline $\mathrm{FeK}_{\alpha}$ Energy $^{f}$ & $6.399_{-0.007}^{+0.006}$ & $6.399_{-0.007}^{+0.006}$ & $\mathrm{keV}$ \\
\hline $\mathrm{FeK}_{\beta}$ Energy $^{f}$ & $7.00 \pm 0.04$ & $7.011_{-0.06}^{+0.04}$ & $\mathrm{keV}$ \\
\hline Nickel Energy ${ }^{f}$ & $7.48 \pm 0.06$ & $7.48_{-0.06}^{+0.05}$ & $\mathrm{keV}$ \\
\hline $\mathrm{FeK}_{\alpha} E W^{g}$ & $0.33_{-0.08}^{+0.11}$ & $0.31_{-0.08}^{+0.10}$ & $\mathrm{keV}$ \\
\hline $\mathrm{FeK}_{\beta} E W^{g}$ & $0.04 \pm 0.01$ & $0.04 \pm 0.01$ & $\mathrm{keV}$ \\
\hline Nickel $E W^{g}$ & $0.09_{-0.05}^{+0.07}$ & $0.11_{-0.06}^{+0.08}$ & $\mathrm{keV}$ \\
\hline \multicolumn{4}{|c|}{ Cold component: $\mathrm{WABS}_{\text {cold }} \times \mathrm{VMEKAL}_{\text {cold }}$} \\
\hline$N_{\text {Hcold }}^{h}$ & $0.07_{-0.06}^{+0.07}$ & $0.10 \pm 0.05$ & $10^{22} \mathrm{~cm}^{-2}$ \\
\hline$k T_{\text {cold }^{i}}^{i}$ & $0.59_{-0.12}^{+0.04}$ & $0.61_{-0.04}^{+0.03}$ & $\mathrm{keV}$ \\
\hline $\mathrm{ab}_{O \text { cold }}^{l}$ & $0.35_{-0.14}^{+0.22}$ & $0.33_{-0.11}^{+0.18}$ & \\
\hline $\mathrm{ab}_{F e \text { cold }}{ }^{l}$ & $0.17_{-0.06}^{+0.09}$ & $0.17_{-0.05}^{+0.08}$ & \\
\hline Norm $_{\text {cold }}{ }^{m}$ & $3.7_{-1.8}^{+3.9}$ & $4.8_{-2.0}^{+2.9}$ & $10^{4} \mathrm{ph} \mathrm{keV}^{-1} \mathrm{~cm}^{-2} \mathrm{~s}^{-1}$ \\
\hline \multicolumn{4}{|c|}{ Medium-temperature component: $\mathrm{WABS}_{\text {med }} \times \mathrm{MEKAL}_{\text {med }}$} \\
\hline$N_{\text {Hmed }}^{h}$ & $0.97_{-0.09}^{+0.18}$ & $1.2 \pm 0.1$ & $10^{22} \mathrm{~cm}^{-2}$ \\
\hline$k T_{\text {med }}^{i}$ & $0.85_{-0.06}^{+0.14}$ & $0.96 \pm 0.06$ & $\mathrm{keV}$ \\
\hline $\mathrm{ab}_{\text {med }}^{l}$ & 1(fixed) & $2.1_{-0.3}^{+0.4}$ & \\
\hline Norm $_{\text {med }}{ }^{m}$ & $6.5_{-1.6}^{+1.4}$ & $6.1_{-1.1}^{+1.3}$ & $10^{4} \mathrm{ph} \mathrm{keV}^{-1} \mathrm{~cm}^{-2} \mathrm{~s}^{-1}$ \\
\hline \multicolumn{3}{|c|}{ Hot component: } & $3 \mathrm{~S}_{\text {hot }} \times$ MEKAL $_{\text {hot }}$ \\
\hline$N_{\text {Hhot }}{ }^{h}$ & $=N_{\mathrm{H} \text { med }}$ & $=N_{\text {Hmed }}$ & $10^{22} \mathrm{~cm}^{-2}$ \\
\hline$k T_{\text {hot }^{i}}^{i}$ & $7.3_{-1.4}^{+1.8}$ & $3.1_{-0.3}^{+0.5}$ & $\mathrm{keV}$ \\
\hline $\mathrm{ab}_{\text {hot }}^{l}$ & $=a b_{\text {med }}$ & $=a b_{\text {med }}$ & \\
\hline Norm $_{\text {hot }}{ }^{m}$ & $7.1 \pm 0.5$ & $6.1 \pm 0.8$ & $10^{4} \mathrm{ph} \mathrm{keV}^{-1} \mathrm{~cm}^{-2} \mathrm{~s}^{-1}$ \\
\hline$\tau_{u \text { hot }^{n}}$ & $4.4_{-0.9}^{+1.4}$ & & $10^{11} \mathrm{~s} \mathrm{~cm}^{-3}$ \\
\hline FPMB/FPMA ${ }^{o}$ & $1.01 \pm 0.03$ & $1.01_{-0.03}^{+0.04}$ & \\
\hline FPMA/PN ${ }^{o}$ & $0.88 \pm 0.04$ & $0.88 \pm 0.04$ & \\
\hline FPMA/MOS ${ }^{o}$ & $0.94_{-0.04}^{+0.05}$ & $0.94_{-0.04}^{+0.05}$ & \\
\hline FPMA/ACIS-S ${ }^{o}$ & $1.00_{-0.04}^{+0.05}$ & $1.00_{-0.04}^{+0.05}$ & \\
\hline$\chi^{2} /$ d.o.f. & $1016.2 / 1035$ & $1028.8 / 1035$ & \\
\hline$\chi_{v}^{2} / \chi^{2}$ prob. & $0.982 / 66 \%$ & $0.994 / 55 \%$ & \\
\hline \multicolumn{4}{|l|}{ Luminosity $^{p}$} \\
\hline plcabs $(2-10 \mathrm{keV})$ & $7.0_{-1.9}^{+2.6}$ & $7.1_{-2.7}^{+1.9}$ & $10^{43} \mathrm{erg} \mathrm{s}^{-1}$ \\
\hline plcabs $(10-40 \mathrm{keV})$ & $8.6_{-2.3}^{+3.3}$ & $7.9_{-3.0}^{+2.1}$ & $10^{43} \mathrm{erg} \mathrm{s}^{-1}$ \\
\hline plcabs $(2-78 \mathrm{keV})$ & $21.8_{-5.7}^{+8.2}$ & $20.5_{-5.4}^{+7.6}$ & $10^{43} \mathrm{erg} \mathrm{s}^{-1}$ \\
\hline pexrav $(2-10 \mathrm{keV})$ & $0.06 \pm 0.02$ & $0.11 \pm 0.02$ & $10^{43} \mathrm{erg} \mathrm{s}^{-1}$ \\
\hline pexrav $(10-40 \mathrm{keV})$ & $0.64 \pm 0.21$ & $1.06_{-0.21}^{+0.19}$ & $10^{43} \mathrm{erg} \mathrm{s}^{-1}$ \\
\hline$k T_{\text {hot }}(2-10 \mathrm{keV})$ & $0.14_{-0.02}^{+0.01}$ & $0.09 \pm 0.01$ & $10^{43} \mathrm{erg} \mathrm{s}^{-1}$ \\
\hline
\end{tabular}

Notes. Best-fit values with uncertainties at the $90 \%$ confidence level for one parameter of interest $\left(\Delta \chi^{2}=2.706\right)$ for model A and B. ${ }^{(a)}$ Fixed Galactic Column density $N_{\mathrm{H}} ;{ }^{(b)}$ column density $N_{\mathrm{H}}$ absorbing the direct power law; ${ }^{(c)}$ direct power-law photon index; ${ }^{(d)}$ normalization at $1 \mathrm{keV}$ of the direct power law; ${ }^{(e)}$ normalization at $1 \mathrm{keV}$ of the reflection component; ${ }^{(f)}$ rest-frame line energy; ${ }^{(g)}$ line equivalent width; ${ }^{(h)}$ column density; ${ }^{(i)}$ plasma temperature; ${ }^{(l)}$ metal abundances; ${ }^{(m)}$ normalization of the thermal components; ${ }^{(n)}$ upper limit on ionization timescales. The subscripts cold, med, hot in $h, i, l, m$, and $n$ indicate the cold, mediumtemperature, and hot thermal components, respectively (see Sect. 4.1). ${ }^{(o)}$ Normalization factors with respect to the FPMA spectrum; ${ }^{(p)}$ intrinsic luminosities. 


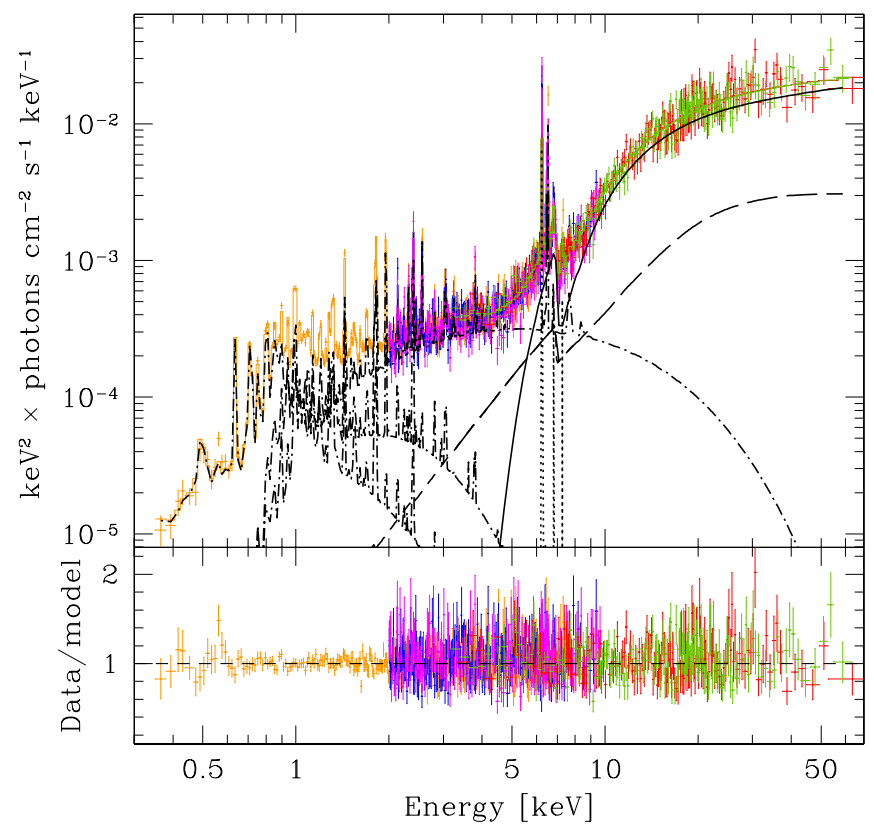

Fig. 8. Broadband spectra of the whole NGC 6240 galaxy, best-fit model (upper panel), and residuals (lower panel). The NuSTAR (green is FPMA, red is FPMB), XMM-Newton (blue is PN, magenta is MOS1+MOS2), and Chandra (orange) spectra unfolded with the instrument responses (for single XMM-Newton and Chandra spectra see Figs. 5 and 4, respectively) are plotted in the upper panel. The total model A is superimposed. The model's single continuum components are also shown: the primary continuum (black solid line); the reflection continuum (black dashed line); the $\mathrm{FeK}_{\alpha}, \mathrm{FeK}_{\beta}$, and nickel lines (black dotted lines); and the three thermal components (black dashdotted lines). The lower panel shows the data/best-fit model ratios.

We note, as pointed out by Murphy \& Yaqoob (2009), that combining PEXRAV and PLCABS may produce a bias toward solutions dominated by the direct continuum. Moreover, a disk/slab geometry with an infinite column density for the material responsible for the Compton-scattered continuum is assumed in the PEXRAV model. The spectral features associated with scattering (e.g., $\mathrm{FeK}_{\alpha}$ and $\mathrm{FeK}_{\beta}$ emission lines and Compton shoulder) in a more realistic, finite column density medium cannot be reproduced by infinite slab models. The MYTORUS model ${ }^{6}$ (Murphy \& Yaqoob 2009; Yaqoob \& Murphy 2011; see also Sect. 5) returns a more physically self-consistent description of the direct continuum and the reflection continuum and lines (Yaqoob \& Murphy 2009, 2011; Yaqoob 2012) and it should not be affected by bias toward transmitted dominated solutions. Nevertheless, the presence in NGC 6240 of two nuclei unresolved by NuSTAR means that this source is not the best case for models like MYTORUS. Therefore, for the whole galaxy we used MYTORUS (described in the following section) for a first-order comparison with the spectral results obtained with model A.

Replacing PLCABS+PEXRAV with MYTORUS in model A, the best-fitting parameters of the absorbed power-law, which describe the nuclear X-ray continuum emission of the two nuclei, are consistent with those obtained using model A (i.e., $\Gamma=1.70 \pm 0.09$ and $\left.N_{\mathrm{H}}=\left(1.39_{-0.08}^{+0.09}\right) \times 10^{24} \mathrm{~cm}^{-2}\right)$. Moreover, the source is still not reflection-dominated; we find that the $10-40 \mathrm{keV}$ reflection component is less than $20 \%$ of the observed direct component. Finally, the nuclear absorption corrected $2-10 \mathrm{keV}$ luminosity is $\sim 8 \times 10^{43} \mathrm{erg} \mathrm{s}^{-1}$, fully consistent with model A.

\footnotetext{
6 http://www . mytorus. com/
}

\section{Resolving the two nuclei}

The NGC 6240 hard X-ray emission is due to two AGNs in the center of the galaxy, resolved by Chandra (Komossa et al. 2003) but not by NuSTAR and XMM-Newton. This is the first attempt to disentangle the primary emission of each nucleus by combining the spatially resolved Chandra spectra with NuSTAR highenergy observations.

\subsection{Chandra spectral analysis of the two individual nuclei}

In order to disentangle the primary emissions, we independently fitted the Chandra spectra of the two nuclei (see Sect. 2.2) in the $0.3-8 \mathrm{keV}$ energy range. The direct and reflection components (continuum and iron line emission) were modeled using the MYTORUS model (Murphy \& Yaqoob 2009; Yaqoob \& Murphy 2011).

Assuming that the direct continuum emission is a power law, three tables are needed to generate spectral fits: MYTORUSZ, MYTORUSS, and MYTORUSL. MYTORUSZ is a multiplicative table that contains the pre-calculated transmission factors that distort the direct continuum at all energies owing to photoelectric absorption and Klein-Nishina scattering (see Sect. 5.2 of the MYTORUS manual). MYTORUSS and MYTORUSL represent the Compton-scattered/reflected continuum toward the line of sight and the fluorescence emission lines typically produced in highly obscured AGNs (i.e., $\mathrm{FeK}_{\alpha}, \mathrm{FeK}_{\beta}$, and Compton shoulder), respectively. We used the MYTORUS model in the original geometry (i.e., the so-called coupled mode, which represents a uniform torus with a half-opening angle of $60^{\circ}$, corresponding to a covering factor of 0.5 ). The normalization of the line component (MYTORUSL) is linked to that of the scattered/reflected continuum (MYTORUSS). A set of MYTORUSL tables are available for a range of energy offsets for best-fitting the peak energies of the emission lines. We found that an offset of $+30 \mathrm{eV}$ is optimal for the northern nucleus. This energy offset is consistent with the results obtained fitting the spectra of the northern and the southern nucleus in the 5.5-8 keV energy range with an oversimplified model (i.e., empirical power-law continuum model plus redshifted Gaussian lines). The $\mathrm{FeK}_{\alpha}$ energy best-fit values are $(6.39 \pm 0.01) \mathrm{keV}$ and $(6.42 \pm 0.01) \mathrm{keV}$ for the southern and northern nucleus, respectively. These values are consistent with those obtained by Wang et al. (2014, see their Table 2). The FeK energy of the northern nucleus line is systematically higher than the theoretical value, the energy blueshift is $20_{-18}^{+14} \mathrm{eV}$ (conservatively, the uncertainties are at the $90 \%$ confidence level for two parameters of interest, i.e., $\Delta \chi^{2}=4.61$ ). This offset could take into account any residual offset due to a blend of neutral and mildly ionized iron. Alternatively, the shift could be the Doppler effect due to outflowing reflecting/absorbing medium with velocity $-940_{-840}^{+660} \mathrm{~km} \mathrm{~s}^{-1}$ (see, e.g., Elvis 2000; Risaliti et al. 2002) or residual relative motion between the two nuclei at the late stage of the merging process (see, e.g., Bournaud et al. 2011). In the latter scenario and assuming the above velocity, the coalescence will be in $\left(0.7_{-0.3}^{+4.6}\right) \mathrm{Myr}$, which is shorter than the timescales of 10-20 Myr estimated by other authors (see, e.g., Scoville et al. 2015).

The spectrum of each nucleus contains part of the X-ray emission of the circumnuclear plasma because the hottest plasma probably originates near the nuclei. Moreover, for projection effects, the spectra can also be contaminated by the coldest plasma. Therefore, we include the multitemperature plasma, modeled using the cold, the medium-temperature, and the shock components as in model A (Sect. 4.2), in the spectral modeling. 
The XSPEC format for the adopted model for each nucleus is

ZPOWERLAW $\times \operatorname{MYTORUSZ}\left(\theta_{\text {obs }}\right)+\operatorname{MYTORUSS}\left(\theta_{\text {obs }}\right)+$ MYTORUSL $\left(\theta_{\text {obs }}\right)+$ WABS $_{\text {cold }} \times$ VMEKAL $_{\text {cold }}+$ WABS $_{\text {med }} \times$ MEKAL $_{\text {med }}+$ WABS $_{\text {hot }} \times$ PSHOCK $_{\text {hot }}$

where $\theta_{\mathrm{obs}}$ is the inclination angle between the torus polar axis and the observer's line of sight and the photon index of the primary continuum was fixed to the best-fit value of the whole galaxy spectrum (see Sect. 4.2 and and Table 3).

We performed a fit initially fixing the parameters describing the temperatures, absorption column densities, and metal abundances of the three thermal components at the best-fit values obtained for the whole galaxy spectrum (see Table 3), leaving the normalizations free to vary. We note that the normalization of the cold thermal component for the northern nucleus is consistent with zero, while for the southern nucleus it is only $\sim 4 \%$ of the normalization of the medium-temperature component. We do not find a statistically good description of the spectra $\left(\chi_{v}^{2} \sim 2\right)$, and some residuals are left at low energies. The best fit for the southern nucleus $\left(\chi_{v}^{2} / \chi^{2}\right.$ prob. $\left.=1.2 / 7 \%\right)$ is obtained with slightly higher values of the temperature of the medium-temperature plasma component and the associated absorbing column density $\left(k T=(1.3 \pm 0.2) \mathrm{keV}, N_{\mathrm{H}}=(1.5 \pm 0.2) \times 10^{22} \mathrm{~cm}^{-2}\right)$, as well as a higher value of the absorbing column density of the shock component $\left(N_{\mathrm{H}}=(10 \pm 3) \times 10^{22} \mathrm{~cm}^{-2}\right)$. This is also the case for the northern nucleus, in which the best-fit $\left(\chi_{v}^{2} / \chi^{2}\right.$ prob. $\left.=1.3 / 3.4 \%\right)$ value of the absorbing column density of the shock component is slightly higher $\left(N_{\mathrm{H}}=(1.9 \pm 0.3) \times 10^{22} \mathrm{~cm}^{-2}\right)$ than that obtained in Sect. 4.2. These results are consistent with the fact that the extended X-ray emission of NGC 6240 over a large scale suffers from blending of thermal plasma with different temperatures, inhomogeneous absorption, and metal abundance. Therefore, spatially resolved analysis in small regions can give results that are slightly different from the results obtained using data from larger regions (e.g., Wang et al. 2014).

The intrinsic 2-10 keV flux due to the shock component is a factor of $\sim 6.5$ larger in the southern nucleus than in the northern, consistent with the scenario that the shock originates from the southern nucleus (see Feruglio et al. 2013b; Wang et al. 2014). We also found that about $60 \%, 10 \%$, and $30 \%$ of the total Fe XXV line flux is emitted in the southern nucleus, northern nucleus, and extended region, respectively. These percentages are consistent with those found by Wang et al. (2014) through spectral and imaging analysis.

The Chandra best-fit parameters of the primary continuum for each nucleus are reported in Table 4. For both nuclei, the inclination angle of the torus and the absorbing column density are not correlated. The inclination angle of the two tori and the absorbing column densities are different above the $99 \%$ confidence level. The equatorial $N_{\mathrm{H}}$ is higher in the northern nucleus, while the inclination angle is larger in the southern one (see Fig. 9). The actual line-of-sight column densities are $N_{\mathrm{H}}=\left(1.47_{-0.17}^{+0.21}\right) \times$ $10^{24} \mathrm{~cm}^{-2}$ and $N_{\mathrm{H}}=\left(1.55_{-0.23}^{+0.72}\right) \times 10^{24} \mathrm{~cm}^{-2}$ for the southern and northern nuclei, respectively. For both nuclei, this is consistent with an optical depth to Thomson scattering of $\tau_{\mathrm{T}} \sim 1.2^{7}$. The equivalent widths of the $\mathrm{FeK}_{\alpha}$ line (Table 4) are fully consistent with previous results (Wang et al. 2014). The intrinsic primary $2-10 \mathrm{keV}$ luminosity of the southern nucleus $\left(5.2 \times 10^{43} \mathrm{erg} \mathrm{s}^{-1}\right)$ is a factor of $\sim 2.6$ larger than that of the northern.

\footnotetext{
$7 \quad \tau_{\mathrm{T}}=x \times \sigma_{\mathrm{T}} \times N_{\mathrm{H}}$, where $\sigma_{\mathrm{T}}$ is the Thomson cross section and $x$ is the mean number of electrons per $\mathrm{H}$ atom, which is $\sim 1.2$ assuming cosmic abundance.
}

Table 4. Chandra best-fit parameters for the two nuclei.

\begin{tabular}{lclc}
\hline \hline Parameter & $S^{a}$ & $N^{b}$ & Units \\
\hline$\Gamma^{c}$ & 1.75 & 1.75 & \\
$N_{\mathrm{H}}{ }^{d}$ & $1.57_{-0.18}^{+0.22}$ & $1.91_{-0.28}^{+0.89}$ & $10^{24} \mathrm{~cm}^{-2}$ \\
$A_{\mathrm{Z}}{ }^{e}$ & $106_{-38}^{+61}$ & $41_{-15}^{+30}$ & $10^{4} \mathrm{ph} \mathrm{keV}^{-1} \mathrm{~cm}^{-2} \mathrm{~s}^{-1}$ \\
$\Theta^{f}$ & $80_{-8}^{+6}$ & $73_{-9}^{+6}$ & $\mathrm{deg}$ \\
$\mathrm{FeK}_{\alpha} E W^{g}$ & $0.37_{-0.05}^{+0.24}$ & $0.58_{-0.09}^{+0.70}$ & $\mathrm{keV}$ \\
$\chi^{2} /$ d.o.f. & $135.2 / 112$ & $90.75 / 68$ & \\
$\chi_{v}{ }^{2} \chi^{2}$ prob. & $1.2 / 6.7 \%$ & $1.34 / 3.4 \%$ & \\
$L_{A_{\mathrm{Z}}}{ }^{h}(2-10 \mathrm{keV})$ & 5.2 & 2.0 & $10^{43} \mathrm{erg} \mathrm{s}^{-1}$ \\
$L_{A_{\mathrm{Z}}}{ }^{i}(10-40 \mathrm{keV})$ & 7.1 & 2.7 & $10^{43} \mathrm{erg} \mathrm{s}^{-1}$ \\
$L_{A_{\mathrm{Z}}}{ }^{i}(2-78 \mathrm{keV})$ & 15.7 & 6.0 & $10^{43} \mathrm{erg} \mathrm{s}^{-1}$ \\
$L_{A_{\mathrm{S}}}{ }^{i}(10-40 \mathrm{keV})$ & 1.2 & 0.5 & $10^{43} \mathrm{erg} \mathrm{s}^{-1}$ \\
\hline
\end{tabular}

Notes. Chandra best-fit values with uncertainties at the $90 \%$ confidence level for one parameter of interest $\left(\Delta \chi^{2}=2.706\right) .{ }^{(a)}$ Best-fit parameters for the southern nucleus; ${ }^{(b)}$ best-fit parameters for the northern nucleus; ${ }^{(c)}$ direct power-law photon index fixed to the best-fit value of the total spectrum (see Sect. 4.2); ${ }^{(d)}$ equatorial column density $N_{\mathrm{H}} ;{ }^{(e)}$ normalization of the primary continuum referred to $1 \mathrm{keV}$; ${ }^{(f)}$ inclination angle between the torus polar axis and the observer's line of sight; ${ }^{(g)}$ line equivalent width; ${ }^{(h)}$ intrinsic primary $2-10 \mathrm{keV}$ luminosities; ${ }^{(i)}$ intrinsic $10-40 \mathrm{keV}$ and 2-78 keV luminosities evaluated extrapolating the Chandra best-fit model using the NuSTAR response efficiency: $L_{A_{Z}}$ for the primary continuum, $L_{A \mathrm{~S}}$ for the reflected/scattered component.

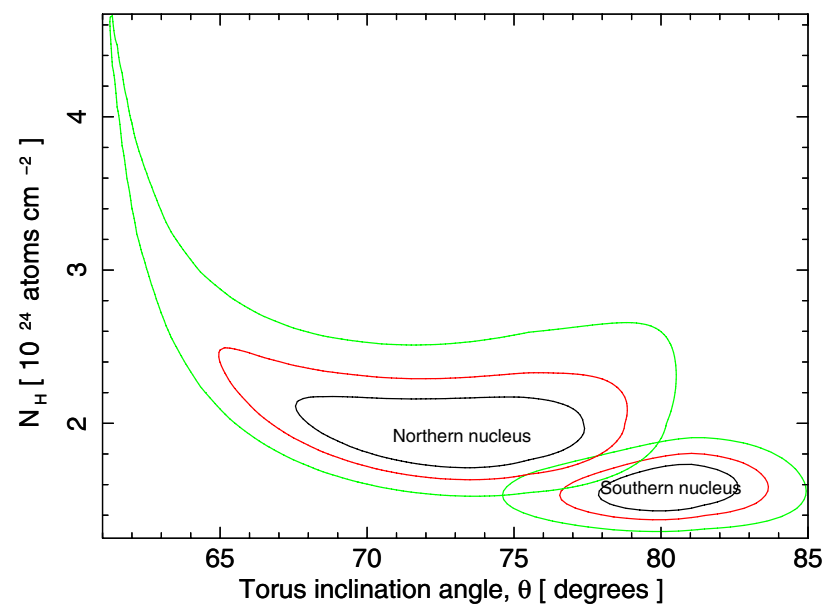

Fig. 9. Chandra $68 \%, 90 \%$, and $99 \%$ confidence contours for the southern and northern nucleus: equatorial column density $N_{\mathrm{H}}$ (in units of $10^{24} \mathrm{~cm}^{-2}$ ) versus $\theta_{\mathrm{obs}}$ (i.e., inclination angle between the torus polar axis and the observer's line of sight). The confidence contours are evaluated leaving $N_{\mathrm{H}}, \theta_{\mathrm{obs}}$, and the normalizations of the direct continuum free to vary.

\subsection{Chandra model of the two nuclei versus the NuSTAR spectrum}

The sum of the best-fit models of the Chandra spectra of the two nuclei described above is extrapolated to high energies and overplotted on the NuSTAR data of the whole galaxy in Fig. 10. A statistically acceptable description of the NuSTAR spectrum is obtained without adjusting the fit: $\chi_{v}^{2} / \chi^{2}$ prob. $=1.16 / 2.7 \%$ for the 3-70 keV energy range, and $1.09 / 15 \%$ ignoring the 7.5-9 keV energy range, where the discrepancies are the largest as judged by the residuals in the lower panel of Fig. 10 . 


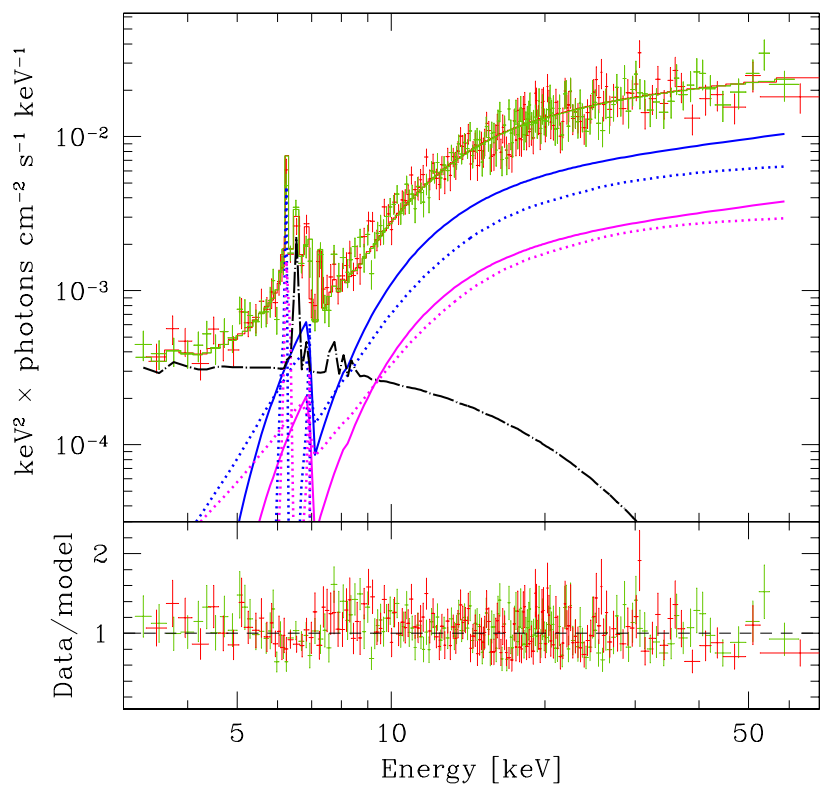

Fig. 10. NuSTAR spectra unfolded with the instrument responses (green is FPMA, red is FPMB) superimposed with the best-fit thermal component of the whole galaxy (black long dash-dotted line, medium-temperature and shock components in model A: WABS $_{\text {med }} \times$ MEKAL $_{\text {med }}+$ WABS $_{\text {hot }} \times$ PSHOCK $_{\text {hot }}$, see Sect. 4.2), and the Chandra best-fit MYTORUS model for the two nuclei (see Sect. 5). The blue lines indicate the primary continuum (solid line) and the reflection continuum and lines (dotted line) of the southern nucleus; the magenta lines are the primary continuum (solid line), the reflection continuum, and lines (dotted line) of the northern nucleus. In the lower panel the NuSTAR data/Chandra best-fit model ratios are shown.

Leaving the normalization of the direct continuum and the equatorial $N_{\mathrm{H}}$ for the southern or the northern nucleus free to vary, the goodness of the fit improves to the $99.8 \%$ confidence level. While the best-fit values of the normalization of the direct continuum remain consistent with those previously measured, the best-fit values of the column density are systematically lower $\left(N_{\mathrm{H}}=(1.38 \pm 0.06) \times 10^{24} \mathrm{~cm}^{-2}\right.$ and $N_{\mathrm{H}}=\left(1.33_{-0.15}^{+0.16}\right) \times 10^{24} \mathrm{~cm}^{-2}$ for the southern and northern nucleus, respectively). This indicates possible column density variations $\leq 2 \times 10^{23} \mathrm{~cm}^{-2}$ on timescales of $\sim 3 \mathrm{yr}$. Fits were also performed leaving the spectral slope free to vary. While for the southern nucleus the best-fit photon index is relatively well constrained $(\Gamma=1.70 \pm 0.08)$, only a lower limit could be obtained for the northern value $(\Gamma>1.67)$. In both cases, these slopes are fully consistent with that obtained fitting the total spectrum $(\Gamma=1.75)$.

\section{Discussion}

The two nuclei in NGC 6240 are separated by $\sim 1^{\prime \prime} .5(\sim 0.7 \mathrm{kpc}$, Fried \& Schultz 1983) and are therefore not spatially resolved by NuSTAR or XMM-Newton. As a first step, we performed joint NuSTAR $+X M M-N e w t o n+$ Chandra broadband spectral analysis $(0.3-70 \mathrm{keV})$ of the data of the whole NGC 6240 galaxy (i.e., the combination of the two nuclei, extended emission, and serendipitous sources), to evaluate the mean properties of the whole system and for comparison with previous high-energy observations.

The broadband $0.3-70 \mathrm{keV}$ spectrum is characterized by three main spectral components (model A in Sect. 4.1 and Table 3). The first is the primary continuum emission piercing through a Compton-thick obscuring medium, which dominates the spectrum at energies $>10 \mathrm{keV}$ where most of the
X-ray luminosity is observed. The second component is the reflected/scattered emission from cold gas, which also generates strong $\mathrm{FeK}_{\alpha}$ and $\mathrm{FeK}_{\beta}$ emission lines at 6-7 keV. The third is a multitemperature thermal plasma due to the nuclear starburst and extended super-wind. Both the transmitted and the reflected components are required by the best fit at a high level of significance $(>5 \sigma)$ according to an F-test. The intensity of the reflection component in the $10-40 \mathrm{keV}$ energy range is on the order of, or slightly lower than, $\sim 20 \%$ of the observed primary emission. Therefore, most of the high-energy flux is transmitted rather than Compton-scattered, and the whole system is not reflection-dominated. This is consistent with the relatively low value of the $\mathrm{FeK}_{\alpha}$ line equivalent width $(\sim 0.3 \mathrm{keV}$; see also Shu et al. 2011; Brightman \& Nandra 2011) and the observed variability at energies $>10 \mathrm{keV}$.

The nuclear emission is characterized by a primary power law continuum with $\Gamma=1.75 \pm 0.08$ and $N_{\mathrm{H}}=\left(1.58_{-0.09}^{+0.10}\right) \times$ $10^{24} \mathrm{~cm}^{-2}$, and intrinsic luminosity of $\left(7.0_{-1.9}^{+2.6}\right) \times 10^{43} \mathrm{erg} \mathrm{s}^{-1}$ and $\left(21.8_{-5.7}^{+8.2}\right) \times 10^{43} \mathrm{erg} \mathrm{s}^{-1}$ in the $2-10 \mathrm{keV}$ and $3-78 \mathrm{keV}$ energy ranges, respectively. Our findings significantly improve upon previous BeppoSAX (Vignati et al. 1999) and RXTE (Ikebe et al. 2000) results, both for disentangling the primary and reflection components, and for better constraining the spectral parameters.

The BeppoSAX best-fit absorbing column density $\left(N_{\mathrm{H}}=\right.$ $\left.\left(2.18_{-0.27}^{+0.30}\right) \times 10^{24} \mathrm{~cm}^{-2}\right)$ is higher than the NuSTAR best-fit value at $>3 \sigma$. To better investigate this discrepancy we re-analyzed the BeppoSAX data using our best-fit model A (see Sect. 4.2 and Table 3) and leaving only the column density obscuring the primary continuum free to vary. We introduced a normalization factor between our best-fit model A and the BeppoSAX data to take into account the flux inter-calibration and the contribution from the serendipitous sources in the MECS extraction region (radius 4', see Sect. 2.4). The best-fit cross-normalization factor is $1.09 \pm 0.05$. We also added a normalization factor between the MECS and PDS data following the ABC BeppoSAX data analysis guide ${ }^{8}$. The best-fit $\left(\chi_{v}^{2} / \chi^{2}\right.$ prob. $\left.=0.84 / 79 \%\right)$ value of the absorbing column density is $N_{\mathrm{H}}=\left(1.82_{-0.10}^{+0.12}\right) \times 10^{24} \mathrm{~cm}^{-2}$, which is lower than, although consistent within the errors with the Vignati et al. (1999) best-fit value. The BeppoSAX bestfit $N_{\mathrm{H}}$ remains systematically higher than that of NuSTAR by about $\sim(1-2) \times 10^{23} \mathrm{~cm}^{-2}$, and the bolometric luminosity is $\sim 9 \%$ higher than that measured from NuSTAR. This luminosity difference includes the inter-calibration uncertainty between the NuSTAR and the BeppoSAX data, and is consistent with little intrinsic variability in NGC 6240. This would also suggest that the BeppoSAX data are not contaminated by bright serendipitous sources.

\subsection{Extended emission}

The NuSTAR data have made a precise measurement of the nuclear continuum of NGC 6240 possible over a broad energy range and in turn allowed better constraints on the softer components associated with the host galaxy. More specifically, the medium-temperature plasma (MEKAL $\mathrm{Med}_{\text {med }}, k T_{\text {med }}$ in Table 3) has an observed luminosity of $2 \times 10^{41} \mathrm{erg} \mathrm{s}^{-1}$ and $\sim 0.9 \times$ $10^{41} \mathrm{erg} \mathrm{s}^{-1}$ in the $0.5-2$ and $2-10 \mathrm{keV}$ energy ranges, respectively. Star-forming galaxies are luminous sources of X-ray emission that originates from $\mathrm{X}$-ray binaries, young supernovae remnants, hot $(0.2-1 \mathrm{keV})$ interstellar gas associated with star-forming regions, and O stars (Fabbiano 1989, 2006

\footnotetext{
8 http://heasarc.nasa.gov/docs/sax/abc/saxabc/saxabc. html
} 
for reviews). Their X-ray luminosity correlates with the SFR (see, e.g., Ranalli et al. 2003; Lehmer et al. 2010 and reference therein), thus using the NGC 6240 SFR $\left(61 M_{\odot} \mathrm{yr}^{-1}\right.$ Yun $\&$ Carilli 2002) and the relations by Ranalli et al. (2003) and Lehmer et al. (2010) we estimate a $0.5-2 \mathrm{keV}$ luminosity of $2.7 \times$ $10^{41} \mathrm{erg} \mathrm{s}^{-1}$ and a $2-10 \mathrm{keV}$ luminosity of $0.7_{-0.4}^{+0.9} \times 10^{41} \mathrm{erg} \mathrm{s}^{-1}$. Despite the uncertainties that affect these relations, these results suggest that the medium-temperature component can be fully due to star formation.

The hot plasma ( $k T_{\text {hot }}$ in Table 3$)$ is fitted with both a nonequilibrium model (model $\mathrm{A}$ in Table 3 ) and an equilibrium model (model $\mathrm{B}$ in Table 3 ). Using model $\mathrm{A}$, the ionization timescale parameter is $\left(4.4_{-0.9}^{+1.4}\right) \times 10^{11} \mathrm{~s} \mathrm{~cm}^{-3}$. If the plasma were close to being in collisional ionization equilibrium then we would expect a value $\geq 10^{12} \mathrm{~s} \mathrm{~cm}^{-3}$ (Masai 1994), suggesting that the plasma is in a marginally nonequilibrium state. The nonequilibrium model is statistically preferred over the equilibrium model $\left(\Delta \chi^{2}=+13\right.$ for the same number of d.o.f.). Furthermore, it does not require super-solar metal abundances, which are required for model $\mathrm{B}\left(\frac{Z}{Z_{\odot}} \sim 2\right)$. The nonequilibrium model strongly favors the presence of a nuclear shock (Feruglio et al. 2013a; Wang et al. 2014).

For the shock component, we evaluated a best-fit value of the column density value of $N_{\mathrm{H}} \simeq 10^{23} \mathrm{~cm}^{-2}$ and an intrinsic $0.5-8 \mathrm{keV}$ luminosity of $\sim 3 \times 10^{42} \mathrm{erg} \mathrm{s}^{-1}$. This value is about a factor of 0.5 smaller than the luminosity obtained from the Chandra spectrum only, but, as noticed by Wang et al. (2014), their absorption-corrected luminosity could be overestimated by a factor of two, given their unlikely large column density of the gas obscuring the hot plasma (i.e., $\left.N_{\mathrm{H}}=(5.5 \pm 1.7) \times 10^{23} \mathrm{~cm}^{-2}\right)$.

\subsection{AGN contribution}

The total AGN bolometric luminosity, calculated assuming the bolometric corrections for type 2 AGNs of Lusso et al. (2011; $\sim 15$ for the observed X-ray luminosity with an uncertainty of a factor of $\sim 3$ ) and the absorption-corrected nuclear $2-10 \mathrm{keV}$ $\mathrm{X}$-ray luminosity is $\left(1.1_{-0.3}^{+0.5}\right) \times 10^{45} \mathrm{erg} \mathrm{s}^{-1}$. This value is a factor of $\sim 2$ lower than that evaluated from the spectral energy distribution (Lira et al. 2002, $L_{\text {bol }} \sim 2 \times 10^{45} \mathrm{erg} \mathrm{s}^{-1}$, assuming the cosmology adopted in this paper). We note that Lira et al. (2002) - extrapolating the best-fit of Vignati et al. (1999) - assumed an AGN unabsorbed $2-10 \mathrm{keV}$ continuum flux of $1 \times 10^{-10} \mathrm{erg} \mathrm{s}^{-1} \mathrm{~cm}^{-2}$, which is $\sim$ twice ours; this explains the discrepancy in the bolometric luminosity. We also note that a lower value of the total AGN bolometric luminosity reduces the discrepancy between the luminosity of the $140 \mathrm{~K}$ component and the absorbed AGN continuum found by the spectral energy distribution (Lira et al. 2002). Therefore, NGC 6240 is one of the most luminous among the local bona fide Compton - Thick AGNs, similar to MRK34 (Gandhi et al. 2014).

Assuming that the 8-1000 $\mu \mathrm{m}$ luminosity of $3.4 \times$ $10^{45} \mathrm{erg} \mathrm{s}^{-1}$ (Wright et al. 1984; Sanders et al. 2003) is a good proxy for the total luminosity, the two AGNs contribute $\sim\left(32_{-9}^{+15}\right) \%$ of the total energy output. This is consistent with the values obtained using multiple methods such as SED+Infrared spectroscopy fitting, PAH features, high- and low-excitation mid-infrared (MIR) lines, and MIR dust continuum (e.g., 20-24\% - Armus et al. 2006; 25-50\% - Lutz et al. 2003; up to $60 \%$ - Egami et al. 2006; $25.8 \%$ - Veilleux et al. 2009; 45-60\% - Mori et al. 2014). The AGN fraction is relatively high for the observed infrared luminosity, but it is not implausible. Alonso-Herrero et al. (2012) showed that in local
LIRGs the AGN bolometric contribution to the IR luminosity of the system is $>25 \%$ in about $8 \%$ of the sample. Moreover, in the local Universe the AGN contribution increases with the IR luminosity of the system (Nardini et al. 2010; Imanishi et al. 2010a,b; Alonso Herrero et al. 2012), and NGC 6240 is a high-luminosity LIRG.

\subsection{Accretion rate of the two nuclei}

The spatially unresolved NuSTAR spectrum is consistent with the sum of the best-fit models obtained from the spectral analysis of the Chandra data of the two nuclei. This suggests that the emission at energies $>10 \mathrm{keV}$ of NGC 6240 is due only to the two nuclei, and is not strongly variable on long timescales $(\sim 3 \mathrm{yr})$. Moreover, there are possible column density variations of $\leq 2 \times 10^{23} \mathrm{~cm}^{-2}$. The two nuclei are both highly absorbed with $\tau_{\mathrm{T}} \sim 1.2$ (i.e., $N_{\mathrm{H}} \sim 1.5 \times 10^{24} \mathrm{~cm}^{-2}$ ) and at energies $>10 \mathrm{keV}$ they are dominated by the primary continuum emission. The actual line-of-sight column density is a few $10^{22} \mathrm{~cm}^{-2}$ larger in the northern nucleus; this would be consistent with the results from the 3-5 $\mu \mathrm{m}$ spectroscopy (Risaliti et al. 2006). The similarity of the column densities of the Compton-thick material obscuring the two nuclei could suggest that there is a common obscurer along the line of sight, possibly originating in gas and dust thrown up by the ongoing galaxy merger. The current data do not allow us to determine where this material lies. The calorimeter on board the Astro- $H$ mission (Takahashi et al. 2014) will allow the profile of the $\mathrm{FeK}_{\alpha}$ to be measured with a resolution on the order of $\approx 350 \mathrm{~km} \mathrm{~s}^{-1}$ and thus it will be possible to determine whether it originates in a compact circumnuclear torus or in larger scale material in the host galaxy (Gandhi et al. 2015).

The intrinsic primary $2-10 \mathrm{keV}$ luminosities of the southern $\left(5.2 \times 10^{43} \mathrm{erg} \mathrm{s}^{-1}\right)$ and northern nucleus $\left(2 \times 10^{43} \mathrm{erg} \mathrm{s}^{-1}\right)$ are fairly consistent (within a factor of $\sim 2$ ) with those expected from the MIR-X-ray luminosity correlation (Gandhi et al. 2009), using the nuclear $12 \mu \mathrm{m}$ fluxes (Asmus et al. 2014).

The bolometric luminosities are $8 \times 10^{44} \mathrm{erg} \mathrm{s}^{-1}$ and $2.6 \times$ $10^{44} \mathrm{erg} \mathrm{s}^{-1}$ for the southern and northern nuclei, respectively, using bolometric corrections for type 2 AGN from Lusso et al. (2011). The southern nucleus is brighter than the northern as expected according to the $3-5 \mu \mathrm{m}$ luminosity (see, e.g., Risaliti et al. 2006; Mori et al. 2014). The bolometric luminosity of the southern nucleus is lower, but consistent with the value from the spectral energy distribution in the near- and mid-IR bands $\left(L_{\text {bol }}=(1.4 \pm 0.6) \times 10^{45} \mathrm{erg} \mathrm{s}^{-1}\right.$, assuming the cosmology adopted in this paper). The slight, not significant, discrepancy could be due to a residual starburst contribution in the MIR flux.

The inferred accretion rate, $\lambda_{\text {Edd }}=L_{\text {bol }} / L_{\text {Edd }}$ is $\sim 0.005_{-0.002}^{+0.003}$ for the southern nucleus, assuming a black hole mass of (0.84-2.2) $\times 10^{9} \quad M_{\odot}$ (Medling et al. 2011), and $\lambda_{\text {Edd }} \sim$ $0.014_{-0.003}^{+0.006}$ for the northern nucleus assuming a black hole mass of $(1.4 \pm 0.4) \times 10^{8} M_{\odot}$ (Engel et al. 2010). The northern nucleus shows a higher $\lambda_{\text {Edd }}$, but the uncertainties on its black hole mass are also a factor of a few. On the contrary, the black hole mass of the southern nucleus is more precise, being determined by highresolution stellar kinematics. The estimated accretion rates are on the low tail of the distribution of Eddington ratios in nearby Seyfert 2 galaxies (e.g., Vasudevan et al. 2010). In this respect, it is similar to the obscured AGNs selected in the GOODS survey (Simmons et al. 2011, 2012). The low value of the accretion rate and the high black hole mass for both nuclei suggests that they could have already assembled most of their mass through accretion processes and are likely to become inactive. The low level 
of accretion in a luminous, highly obscured early merger system is somewhat unexpected. The relatively low accretion rate may be associated with the strong molecular outflow (Feruglio et al. 2013b), which has depleted the nuclear regions of gas. We also note that in spite of the large uncertainties in the black hole mass and Eddington ratio, the photon index of the primary continuum is consistent with the low accretion rates of the two nuclei according to the relation found for a few AGN type 1 samples (Shemmer et al. 2008; Risaliti et al. 2009; Brightman et al. 2013).

\subsection{Hard X-ray variability}

A detailed analysis of the light curves in several independent energy bands clearly reveals hard X-ray variability on timescales of $\sim 15-20 \mathrm{ks}$ by up to $\sim 20 \%$. The variability peaks at $\sim 30 \mathrm{keV}$ $(\sim 40 \%)$. These results are consistent with the variability detected earlier in the Swift BAT data on substantially longer timescales (i.e., months/years). In fact, Soldi et al. (2014) found maximum $(54 \pm 18 \%)$ and minimum variability $(4 \pm 3 \%)$ in the $14-24$ and 35-100 keV energy bands, respectively. Moreover, by reanalyzing the BeppoSAX PDS data $(\sim 15-200 \mathrm{keV})$, we found variability up to $\sim 50 \%$ on timescales fully consistent with those of the NuSTAR hard X-ray light curve. The PDS has a field of view (FOV) of $1.3^{\circ}$ and therefore the data could suffer from contamination. Nevertheless, for NGC 6240, no known bright sources are present in the PDS FOV, and the probability of a serendipitous source in the PDS FOV with a flux equal to or larger than that of NGC 6240 is $\leq 10^{-5}$ (see Vignati et al. 1999).

Assuming that the NuSTAR, BeppoSAX PDS, and Swift BAT hard X-ray variability have the same origin, the observed variability can be explained by the two following hypotheses:

a) the variability is due to flux variations of the transmitted component of the southern nucleus up to $\sim 20-30 \%$ for NuSTAR, and by $\sim 50-70 \%$ for the BeppoSAX PDS and Swift BAT observed variability. Indeed, the lack of variability of the $\mathrm{FeK}_{\alpha}$ line suggests that the reflection component is constant. Moreover, the spectral analysis suggests that the nuclei are transmission dominated at energies $>10 \mathrm{keV}$.

b) On the contrary, if the variability is due to the northern nucleus, the amplitude of the variations should be up to $\sim 50-60 \%$ for NuSTAR, and up to a factor of $\sim 2.6$ for the BeppoSAX PDS and Swift BAT observed variability. The NuSTAR variability would be consistent with the tentative $\sim 30 \%$ variability in the $6.4-8 \mathrm{keV}$ Chandra light curve of the northern nucleus. Nevertheless the NuSTAR, BeppoSAX PDS, and Swift BAT hard X-ray variability would imply that the northern nucleus should be as bright as, or brighter than the southern nucleus in some periods. This is difficult to reconcile with the evidence that over the last $\sim 15 \mathrm{yr}$ all the data suggest that the southern nucleus has been brighter than the northern as indicated by the Chandra data (Komossa et al. 2003; Shu et al. 2011; Wang et al. 2014) and 3-5 $\mu \mathrm{m} \mathrm{lu-}$ minosity (see, e.g., Mori et al. 2014; Risaliti et al. 2006). Therefore, the most plausible hypothesis is that the hard $\mathrm{X}$-ray variability is mainly due to variations of the transmitted component of the southern nucleus.

The $6.4-8 \mathrm{keV}$ tentative variability of the northern nucleus suggests changes of the direct continuum and/or of the column density; we are not able to disentangle them with the current data. In all cases, the different $6.4-8 \mathrm{keV}$ variability could be an indication that the matter reflecting/Compton-scattering the nuclear emission is distributed differently in the nuclei, as the results obtained in the present analysis seem to suggest (see Fig. 9).

On timescales on the order of years, the mean hard X-ray flux state is approximately constant, suggesting that the accretion rates of the two nuclei do not experience strong variability on year-long timescales.

The variability observed in NGC 6240 is similar to that observed in NGC 4945. In NGC 4945 the hard X-ray variability is also ascribed to changes of the primary continuum, confirmed through a detailed count rate resolved spectral analysis (Puccetti et al. 2014). In NGC 4945, the fast and large hard X-ray variability, the constancy of the $\mathrm{FeK}_{\alpha}$ line, and the huge Compton depth along the line of sight $\left(\tau_{\mathrm{T}} \sim 2.9\right)$ suggest a low global covering factor $(\sim 0.15)$ for the circumnuclear gas. In comparison with NGC 4945, in NGC 6240 the $\mathrm{FeK}_{\alpha}$ line is constant, but the hard X-ray variability is smaller and slower, likely due to the higher black hole masses and lower accretion rates, and the Compton depth along the line of sight is a factor $\sim 2.5$ lower. These findings indicate that the global covering factor for NGC 6240 should be larger than in NGC 4945.

\section{Conclusion}

We present NuSTAR observations of NGC 6240, one of the nearest luminous infrared galaxies in a relatively early merger state with two distinct nuclei separated by a distance of $\sim 1$ '. 5 . The main results of this work can be summarized as follows:

- We clearly detect at a high significance $(>5 \sigma)$ both the transmitted primary absorbed continuum and the cold reflection continuum. The primary continuum is obscured by a $N_{\mathrm{H}}$ of $\sim 1.6 \times 10^{24} \mathrm{~cm}^{-2}$ and dominates at energies $>10 \mathrm{keV}$.

- The total AGN bolometric luminosity is $\left(1.1_{-0.3}^{+0.5}\right) \times 10^{45} \mathrm{erg} \mathrm{s}^{-1}$, which is $\left(32_{-9}^{+15}\right) \%$ of the infrared luminosity. This indicates that the galaxy emission is not dominated by the two AGNs, but likely by a starburst and related processes.

- The spatially unresolved NuSTAR hard X-ray spectrum of NGC 6240 is consistent with the sum of the Chandra bestfit models of the two nuclei extrapolated to the NuSTAR hard X-ray energies. This suggests that the hard emission of NGC 6240 is only due to the two nuclei, and that it has not varied by much on a decade-long timescale. Both nuclei are highly absorbed with $\tau_{\mathrm{T}} \sim 1.2$ (i.e., $N_{\mathrm{H}} \sim 1.5 \times 10^{24} \mathrm{~cm}^{-2}$ ) as expected in the early stage of a major merger, and have bolometric luminosities $\sim 8 \times 10^{44} \mathrm{erg} \mathrm{s}^{-1}$ (southern nucleus) and $\sim 2.6 \times 10^{44} \mathrm{erg} \mathrm{s}^{-1}$ (northern nucleus). The two nuclei show low accretion rates $\left(\lambda_{\text {Edd }}=L_{\text {bol }} / L_{\text {Edd }}\right.$ is $\sim 0.005_{-0.002}^{+0.003}$ for the southern nucleus and $\lambda_{\text {Edd }} \sim 0.014_{-0.003}^{+0.006}$ for the northern nucleus).

- We found variability in the NuSTAR light curve at energies $>10 \mathrm{keV}$ of $\sim 20 \%$ on timescales of $15-20 \mathrm{ks}$. We found similar variability in the BeppoSAX PDS light curve. Various arguments lead us to conclude that the hard X-ray variability is due to variations of the primary continuum of the southern nucleus.

- Finally, by comparing NuSTAR, BeppoSAX, and Chandra archival data we found that the two nuclei remain consistently Compton-thick. Although we find evidence of variability of the material along the line of sight with column densities $N_{\mathrm{H}} \leq 2 \times 10^{23}$ on timescales of $\sim 3-15 \mathrm{yr}$.

Variability below $10 \mathrm{keV}$ is not observed in Compton-thick AGNs because of absorption and reflection. Above $10 \mathrm{keV}$ 
only a few sources have been sampled with sufficient statistics, and NuSTAR is finding variability to be more common (NGC 4945, Puccetti et al. 2014; NGC 1068, Marinucci et al. 2016; NGC 6240, this work). Therefore, to investigate variability in this type of source, good sampling of the 30-50 keV energy range is mandatory. Further NuSTAR monitoring of NGC 6240 would allow a detailed time/count rate resolved spectral analysis and would help put constraints on the geometry/location of the reflecting/absorbing medium.

Acknowledgements. This work was supported under NASA Contract NNG08FD60C, and made use of data from the NuSTAR mission, a project led by the California Institute of Technology, managed by the Jet Propulsion Laboratory, and funded by the National Aeronautics and Space Administration. We thank the NuSTAR Operations, Software and Calibration teams for support with the execution and analysis of these observations. This research has made use of the NuSTAR Data Analysis Software (NuSTARDAS) jointly developed by the ASI Science Data Center (ASDC, Italy) and the California Institute of Technology (USA). A.C., A.M., F.F. and L.Z. acknowledge support from the ASI/INAF grant I/037/12/0 - 011/13. W.N.B. acknowledges support from Caltech NuSTAR sub-contract 44A-1092750. F.E.B. and C.R. acknowledge support from CONICYT-Chile grants Basal-CATA PFB-06/2007 and FONDECYT 1141218. F.E.B., C.R. and P.A. acknowledge support from "EMBIGGEN" Anillo ACT1101. FEB acknowledges support from the Ministry of Economy, Development, and Tourism's Millennium Science Initiative through grant IC120009, awarded to The Millennium Institute of Astrophysics, MAS. M.B. acknowledges support from NASA Headquarters under the NASA Earth and Space Science Fellowship Program, grant NNX14AQ07H.

\section{References}

Alonso-Herrero, A., Pereira-Santaella, M., Rieke, G. H., \& Rigopoulou, D. 2012, ApJ, 744, 2

Anders, E., \& Grevesse, N. 1989, Geochim. Cosmochim. Acta 53, 197

Arévalo, P., Bauer, F. E., Puccetti, S., et al. 2014, ApJ, 791, 81

Armus, L., Bernard-Salas, J., Spoon, H. W. W., et al. 2006, ApJ, 640, 204

Asmus, D., Hönig, S. F., Gandhi, P., Smette, A., \& Duschl, W. J. 2014, MNRAS, 439, 1648

Bauer, F. E., Arévalo, P., Walton, D. J., et al. 2015, ApJ, 812, 116

Boella, G., Butler, R. C., Perola, G. C., et al. 1997, A\&AS, 122, 299

Boller, Th., Keil, R., Hasinger, G., et al. 2003, A\&A, 411, 63

Bournaud, F., Chapon, D., Teyssier, R., et al. 2011, ApJ, 730, 4

Brightman, M., \& Nandra, K. 2011, MNRAS, 413, 1206

Brightman, M., Silverman, J. D., Mainieri, V., et al. 2013, MNRAS, 433, 2485

Bush, S. J., Wang, Z., Karovska, M., \& Fazio, G. G. 2008, ApJ, 688, 875

Condon, J. J., Condon, M. A., Gisler, G., \& Puschell, J. J. 1982, ApJ, 252, 102

Di Matteo, T., Springel, V., \& Hernquist, L. 2005, Nature, 433, 604

Downes, D., Solomon, P. M., \& Radford, S. J. E. 1993, ApJ, 414, L13

Egami, E., Neugebauer, G., Soifer, B. T., et al. 2006, AJ, 131, 1253

Elvis, M. 2000, ApJ, 545, 63

Engel, H., Davies, R. I., Genzel, R., et al. 2010, A\&A, 524, A56

Fabbiano, G. 1989, ARA\&A, 27, 87

Fabbiano, G. 2006, ARA\&A, 44, 323

Feruglio, C., Fiore, F., Maiolino, R., et al. 2013a, A\&A, 549, A51

Feruglio, C., Fiore, F., Piconcelli, E., et al. 2013b, A\&A, 558, A8

Fosbury, R. A. E., \& Wall, J. V. 1979, MNRAS, 189, 79

Fried, J. W., \& Schulz, H. 1983, A\&A, 118, 166

Frontera, F., Costa, E., dal Fiume, D., et al. 1997, SPIE, 3114, 206

Fruscione, A., McDowell, J. C., Allen, G. E., et al. 2006, Proc. SPIE, 6270

Gandhi, P., Horst, H., Smette, A., et al. 2009, A\&A, 502, 457

Gandhi, P., Lansbury, G. B., Alexander, D. M., et al. 2014, ApJ, 792, 117

Gandhi, P., Hoenig, S. F., \& Kishimoto, M. 2015, ApJ, 812, 113

Harrison, F. A., Craig, William, W., et al. 2013, ApJ, 770, 103

Heckman, T. M., Armus, L., \& Miley, G. K. 1987, AJ, 92, 276
Hopkins, P. F., \& Elvis, M. 2010, MNRAS, 401, 7

Ikebe, Y., Leighly, K., Tanaka, Y., et al. 2000, MNRAS, 316, 433 Imanishi, M., Maiolino, R., \& Nakagawa, T. 2010a, ApJ, 709, 80

Imanishi, M., Nakagawa, T., Shirahata, M., Ohyama, Y., \& Onaka, T. 2010b, ApJ, 21, 1233

Iwasawa, K., Comastri, A. 1998, MNRAS, 297, 1219

Kalberla, P. M. W., Burton, W. B., Hartmann, D. A., et al. 2005, A\&A, 440, 775

Keel, W. C. 1990, AJ, 100, 356

Komossa, S., Schulz, H., Greiner, J., et al. 1998, A\&A, 334, 110

Komossa, S., Burwitz, V., Hasinger, G., et al. 2003, ApJ, 582, L15

Lehmer, B. D., Alexander, D. M., Bauer, F. E., et al. 2010, ApJ, 724, 559

Lira, P., Ward, M. J., Zezas, A., \& Murray, S. S. 2002, MNRAS, 333, 709

Lusso, E., Comastri, A., Vignali, C., et al. 2011, A\&A, 534, A110

Lutz, D., Sturm, E., Genzel, R., et al. 2003, A\&A, 409, 867

Magdziarz, P., \& Zdziarski, A. A. 1995, MNRAS, 273, 837

Marinucci, A., Bianchi, S., Matt, G., et al. 2016, MNRAS, 456, L94

Masai, K. 1994, ApJ, 437, 770

Medling, A. M., Ammons, S. M., Max, C. E., et al. 2011, ApJ, 743, 32

Mori, T. I., Imanishi, M., Alonso-Herrero, A., et al. 2014, PASJ, 66, 93

Morris, S. L., \& Ward, M. J. 1988, MNRAS, 230, 639

Murphy, K. D., \& Yaqoob, T. 2009, MNRAS, 397, 1549

Nardini, E., Risaliti, G., Watabe, Y., Salvati, M., \& Sani, E. 2010, MNRAS, 405, 2505

Nardini, E., Wang, J., Fabbiano, G., et al. 2013, ApJ, 765, 141

Netzer, H., Lemze, D., Kaspi, S., et al. 2005, ApJ, 629, 739

Palmeri, P., Mendoza, C., Kallman, T. R., Bautista, M. A., \& Meléndez, M. 2003, A\&A, 410, 359

Planck Collaboration XVI. 2014, A\&A, 571, A16

Puccetti, S., Fiore, F., Vignali, C., et al. 2009, ApJS, 185, 586

Puccetti, S., Comastri, A., Fiore, F., et al. 2014, ApJ, 793, 26

Ranalli, P., Comastri, A., \& Setti, G. 2003, A\&A, 399, 39

Rafanelli, P., Schulz, H., Barbieri, C., et al. 1997, A\&A, 327, 901

Risaliti, G., Elvis, M., \& Nicastro, F. 2002, ApJ, 571, 234

Risaliti, G., Elvis, M., Fabbiano, G., Baldi, A., \& Zezas, A. 2005, ApJ, 623, L93

Risaliti, G., Sani, E., Maiolino, R., et al. 2006, ApJ, 637, L17

Risaliti, G., Elvis, M., Fabbiano, G., et al. 2007, ApJ, 659, L111

Risaliti, G., Young, M., \& Elvis, M. 2009, ApJ, 700, L6

Sanders, D. B., Mazzarella, J. M., Kim, D. C., et al. 2003, AJ, 126, 1607

Santini, P., Fontana, A., Grazian, A., et al. 2009, A\&A, 504, 751

Schmitt, H. R., Bica, E., \& Pastoriza, M. G. 1996, MNRAS, 278, 965

Scoville, N., Sheth, K., Walter, F., et al. 2015, ApJ, 800, 70

Shemmer, O., Brandt, W. N., Netzer, H., Maiolino, R., \& Kaspi, S. 2008, ApJ, 682,81

Shu, X. W., Yaqoob, T., \& Wang, J. X. 2011, ApJ, 738, 147

Simmons, B. D., Van Duyne, J., Urry, C. M., et al. 2011, ApJ, 734, 121

Simmons, B. D., Urry, C. M., Schawinski, K., Cardamone, C., \& Glikman, E. 2012, ApJ, 761, 75

Soldi, S., Beckmann, V., Baumgartner, W. H., et al. 2014, A\&A, 563, A57

Springel, V., White, S. D. M., Jenkins, A., et al. 2005, Nature, 435, 629

Tacconi, L. J., Genzel, R., Tecza, M., et al. 1999, ApJ, 524, 732

Takahashi, T., Mitsuda, K., Kelley, R., et al. 2014, SPIE, 9144, 24

Tecza, M., Genzel, R., Tacconi, L. J., et al. 2000, ApJ, 537, 178

Tremaine, S., Gebhardt, K., Bender, R., et al. 2002, ApJ, 574, 740

Vasudevan, R. V., Fabian, A. C., Gandhi, P., Winter, L. M., \& Mushotzky, R. F. 2010, MNRAS, 402, 1081

Vignati, P., Molendi, S., Matt, G., et al. 1999, A\&A, 349, L57

Veilleux, S., Kim, D.-C., Sanders, D. B., Mazzarella, J. M., \& Soifer, B. T. 1995, ApJS, 98, 171

Veilleux, S., Rupke, D. S. N., Kim, D.-C., et al. 2009, ApJS, 182, 628

Veilleux, S., Meléndez, M., Sturm, E., et al. 2013, ApJ, 776, 27

Walton, D. J., Middleton, M. J., Rana, V., et al. 2015, ApJ, 806, 65

Wang, J., Nardini, E., Fabbiano, G., et al. 2014, ApJ, 781, 55

Wright, G. S., Joseph, R. D., \& Meikle, W. P. S. 1984, Nature, 309, 430

Yaqoob, T. 1997, ApJ, 479, 184

Yaqoob, T., \& Murphy, K. D. 2011, MNRAS, 412, 1765

Yaqoob, T. 2012, MNRAS, 423, 3360

Yun, M. S., \& Carilli, C. L. 2002, ApJ, 568, 88

Zasov, A. V., \& Karachentsev, I. D. 1979, Sov. Astron. Lett., 5, 126 